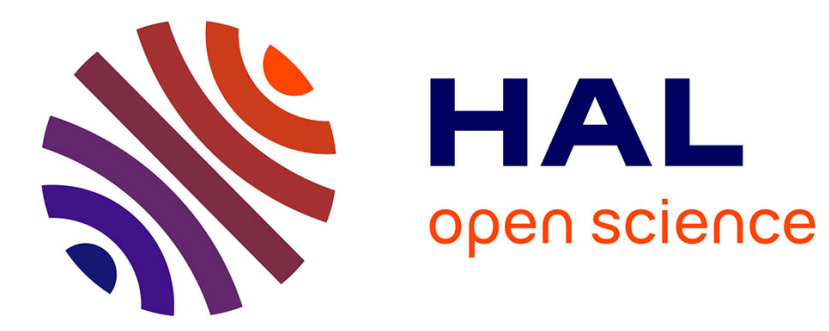

\title{
Semiclassical initial value representation: From Møller to Miller
}

Laurent Bonnet

\section{To cite this version:}

Laurent Bonnet. Semiclassical initial value representation: From Møller to Miller. Journal of Chemical Physics, 2020, 153 (17), pp.174102. 10.1063/5.0023137 . hal-03372235

\section{HAL Id: hal-03372235 \\ https://hal.science/hal-03372235}

Submitted on 10 Oct 2021

HAL is a multi-disciplinary open access archive for the deposit and dissemination of scientific research documents, whether they are published or not. The documents may come from teaching and research institutions in France or abroad, or from public or private research centers.
L'archive ouverte pluridisciplinaire HAL, est destinée au dépôt et à la diffusion de documents scientifiques de niveau recherche, publiés ou non, émanant des établissements d'enseignement et de recherche français ou étrangers, des laboratoires publics ou privés. 


\title{
Semiclassical initial value representation:
} From Møller to Miller

\author{
L. Bonnet ${ }^{*}, \dagger$ \\ † CNRS, ISM, UMR 5255, F-33400 Talence, France \\ † Université de Bordeaux, ISM, UMR 5255, F-33400 Talence, France \\ E-mail: claude-laurent.bonnet@u-bordeaux.fr
}




\begin{abstract}
The initial value representation (IVR) was proposed five decades ago by W. H. Miller in order to improve the feasibility and accuracy of semiclassical (SC) scattering calculations [J. Chem. Phys. 53, 3578 (1970)]. Møller operators, which play a fundamental role in quantum scattering theory, do not appear in his formulation based on action-angle coordinates. These operators were introduced much later by Garashchuk and Light in SC-IVR calculations [J. Chem. Phys. 114, 1060 (2001)] performed in Cartesian coordinates within the Tannor and Weeks formulation of quantum scattering theory [J. Chem. Phys. 98, 3884 (1993)]. Remarkably, Møller operators were found to boost the numerical efficiency of SC-IVR calculations. The purpose of this work is to show within a simple model of light-induced rotational transitions that in fact, Møller operators were already underlying Miller's pioneering formulation. In line with the results of Garashchuk and Light, removing the action of these operators in Miller's theory strongly decreases its numerical efficiency.
\end{abstract}

\title{
1 Introduction
}

Semiclassical mechanics aims at reproducing quantum mechanical effects by assigning probability amplitudes to classical paths and making them interfere. Since the concept of classical path is among the most intuitive ones, the interpretative power of semiclassical mechanics, whenever applicable, is unmatched. ${ }^{1-6}$ We focus in this work on its application to scattering processes, though it also finds a wide range of application in spectroscopy. ${ }^{4-7}$

In the general case where resonances are involved in the dynamics, reasonably accurate semiclassical (SC) calculations for processes of experimental interest are still very hard to perform despite major efforts to improve their numerical efficiencies. ${ }^{8,9}$ Nevertheless, the semiclassical approach remains the best guide for introducing quantum corrections in the classical treatment of the dynamics, or justifying ad hoc corrections to the latter based on intuitive grounds. ${ }^{10}$ This is of great importance for the study of multidimensional systems, ${ }^{11}$ or 
the physical interpretation of quantum scattering results. ${ }^{10,12}$ Improving our understanding of the semiclassical description is thus highly desirable.

The $S$ matrix is a central object of collision theory. ${ }^{5,13}$ One route to the estimation of its elements in terms of classical trajectories is as follows. First, one expresses the elements in terms of Feynman space-time propagator. ${ }^{1}$ Second, one replaces the latter by its standard semiclassical limit developped by van Vleck and Gutzwiller (VVG). ${ }^{4,8} S$ matrix elements are then given by a multidimensional integral with respect to initial and final configuration space coordinates, and time. This integral can be calculated either analytically within the stationary phase approximation (SPA), ${ }^{4}$ or numerically, or within the combination of both methods for two complementary subsets of variables. The idea behind the SPA is the following: the integrand is a complex number the phase of which rapidly varies when moving away from its stationary points. Therefore, the integrand strongly oscillates everywhere but in the neighborhoods of the stationary points which, consequently, mainly contribute to the integral. The SPA assumes that the second order development of the phase is valid over these neighborhoods, thus allowing to apply standard results of complex analysis to analytically perform the integration ${ }^{4}$ (see also Eqs. (A.11) and (28) in this work for one and two dimensions, respectively). If all the variables are integrated within the SPA, the resulting expression is the celebrated formula of classical $S$ matrix theory (CSMT). ${ }^{1}$ Being the most classical SC approach, CSMT is also known as the classical-limit quantum mechanical theory of molecular collisions. ${ }^{1}$ The advantages of this approach are elegance and explanatory strength. However, CSMT requires the search for trajectories connecting integer actions in the infinite past and future. Unfortunately, this root search is a daunting task for most real life processes, thus, preventing CSMT from being generally applicable. As stated above, the alternative is to integrate within the SPA over a subset of variables and numerically over the remaining variables, or to perform a fully numerical integration; this is where the initial value representation (IVR) comes into play. ${ }^{8}$ Briefly, the IVR is based on the following trick: one uses the SPA to get rid of the final space coordinates while fixing the values of the initial 
momenta, or one just replaces the final space coordinates by the initial momenta. More details will be given further on.

IVR was originally introduced by W. H. Miller in his semiclassical formulation of collinear atom-diatom inelastic collisions in action-angle coordinates for the vibrational degree-offreedom. ${ }^{14}$ In this approach, the coordinates of the problem are the vibrational phase $q$ of the diatom and the distance $R$ between its center-of-mass and the atom. $P$ is the momentum conjugate to $R$. $\mu$ is the reduced mass of the atom with respect to the diatom and $\omega$ is its streching frequency. The $n^{t h}$ vibrational state is approximated by $\langle q \mid n\rangle=e^{i n q} / \sqrt{2 \pi}$ (note that if $q$ and $n$ were, respectively, the orientation angle of a planar rotor and its rotational quantum number, the previous state would be rigorously exact). $S$ matrix elements are obtained by projecting the final and initial states $\left\langle n_{2}\right|$ and $\left|n_{1}\right\rangle$ on the time Fourier transform of the evolution operator. ${ }^{1}$ One might thus expect to obtain $S$ matrix elements as an integral over initial and final values $q_{1}$ and $q_{2}$ of $q$, called natural angle in the following. However, for subtle reasons detailed in Ref. ${ }^{14}$, Miller's original IVR involves $S$ matrix elements given by an integral over initial and final values of a different angle $\bar{q}$ shifted with respect to $q$ according to

$$
\bar{q}=q-\omega \frac{\mu R}{P}
$$

$\bar{q}$ will be called shifted angle thereafter (see Ref. ${ }^{15}$ for the use of this variable in classical mechanics). Since $P=\mu \dot{R}$, the absolute value of $\mu R / P=R / \dot{R}$ is the time to run the distance $R$ when ignoring the interaction between the atom and the diatom. $\mu R / P$ has the sign of $P$. It is thus negative (positive) if the system is incoming (outgoing). Calling $\left(R_{1}, P_{1}\right)$ and $\left(R_{2}, P_{2}\right)$ the initial and final values of $(R, P)$ and $t_{1}$ and $t_{2}$ the absolute values of $\mu R_{1} / P_{1}$ and $\mu R_{2} / P_{2}$, we can rewrite Eq. (1) at $R_{1}$ and $R_{2}$ as

$$
q_{1}=\bar{q}_{1}-\omega t_{1}
$$


and

$$
\bar{q}_{2}=q_{2}-\omega t_{2} .
$$

These equations can be interpreted as follows. A trajectory is started at $R=0$ with the negative momentum $P_{1}$ and $q=\bar{q}_{1}$ and is run backward in time during $t_{1}$ with the free classical Hamiltonian $H_{0}$, i.e., without the interaction potential. This substracts $\omega t_{1}$ to $\bar{q}_{1}$ and leads to $q_{1}$ (Eq. (2)) and $R=R_{1}$. One then runs the trajectory forward in time with the full classical Hamiltonian $H$ from $\left(R_{1}, q_{1}\right)$ to $\left(R_{2}, q_{2}\right)$. This step is the usual one in standard classical trajectory calculations. Finally, the trajectory is run backward in time with $H_{0}$ during $t_{2}$, i.e., from $\left(R_{2}, q_{2}\right)$ to $\left(R=0, \bar{q}_{2}\right)$. This substracts $\omega t_{2}$ to $q_{2}$ (Eq. (3)). The backward run with $H_{0}$ from time 0 to $-t_{1}$ and the begining of the forward run with $H$ from $-t_{1}$ to time 0 is the classical analog of Møller operator $\Omega_{+}{ }^{5,13}$ (see further below). The previous three step trajectory, however, can be run in the reverse direction. When doing so, the forward run with $H_{0}$ from $\left(R=0, \bar{q}_{2}\right)$ at time 0 to $\left(R_{2}, q_{2}\right)$ at $t_{2}$, and the begining of the backward run with $H$ from $t_{2}$ to time 0 is the classical analog of Møller operator $\Omega_{-}{ }^{5,13}$ Despite this analogy, it does not seem that the formal link between Miller's original SCIVR expression (Eq. (29) in Ref. ${ }^{14}$ ) and Møller operators has ever been established. Since these operators play a fundamental role in quantum scattering theory, ${ }^{5,13}$ the goal of the present work is to shed light on this link within a simple model of light-induced rotational transitions. ${ }^{16}$

On the other hand, one cannot say that Møller operators have never been involved in semiclassical calculations. They were indeed explicitely introduced two decades ago by Garashchuk and Light ${ }^{17}$ in SC-IVR calculations performed in Cartesian coordinates within the Tannor and Weeks formulation of quantum scattering theory. ${ }^{18}$ The process under study was the collinear $\mathrm{H}+\mathrm{H}_{2}$ reaction. Ten times less trajectories were found to be necessary for the same level of accuracy as calculations made without Møller operators. We show here that the same happens with Miller's original formulation: deriving it in terms of natural angles instead of shifted ones, which as we will see amounts to ignore Møller operators, strongly 
decreases its numerical efficiency. Worse than this, this strongly deteriorates the accuracy of its predictions on rotational transitions.

\section{Møller operators and representation in shifted angles}

\section{$2.1 \quad$ Møller operators}

We consider a rigid diatom of moment of inertia $I$ interacting with a laser pulse inducing rotational transitions. The rotor is supposed to be planar, i.e., rotating within a fixed plane of the laboratory frame. This restriction makes the mathematical developments much less involved. Before and after the interaction with the pulse, which takes place around time $\tau$ equal 0 , the diatom rotates freely. $\phi$ and $J$ are, respectively, the orientation angle of the diatom and its conjugate angular momentum. The Hamiltonian of the system is

$$
H=H_{0}+V
$$

where

$$
H_{0}=-\frac{1}{2 I} \frac{\partial^{2}}{\partial \phi^{2}}
$$

is the Hamiltonian of the free rigid diatom ( $\hbar$ units are used throughout this work) and $V$ is the operator associated with $V(\phi, \tau)$, the time dependent angular potential created by the laser pulse. We define the stationary states of $H_{0}$ at time $\tau$ by $|j ; \tau\rangle$ where $j$ is the rotational

quantum number. The projection of $|j ; 0\rangle$ on $|\phi\rangle$ is set at

$$
\langle\phi \mid j ; 0\rangle=\frac{e^{i j \phi}}{(2 \pi)^{1 / 2}}
$$

and $\langle\phi \mid j ; \tau\rangle$ is straightforwardly deduced from $\langle\phi \mid j ; 0\rangle$ by the relation

$$
|j ; \tau\rangle=e^{-i H_{0} \tau}|j ; 0\rangle=e^{-i E_{j} \tau}|j ; 0\rangle
$$


where

$$
E_{j}=\frac{j^{2}}{2 I}
$$

We wish to semiclassically calculate the probability amplitude $S_{j_{2} j_{1}}$ that the diatom, in the rotational state $j_{1}$ at $\tau=-\infty$, ends up in the rotational state $j_{2}$ at $\tau=+\infty$. This $S$ matrix element is given by

$$
S_{j_{2} j_{1}}=\left\langle j_{2} ; t\left|e^{-i H 2 t}\right| j_{1} ;-t\right\rangle
$$

where $t$ is a value of $\tau$ tending to $+\infty . S_{j_{2} j_{1}}$ can be rewritten as

$$
S_{j_{2} j_{1}}=\left\langle j_{2} ; t\left|e^{-i H_{0} t} e^{i H_{0} t} e^{-i H 2 t} e^{i H_{0} t} e^{-i H_{0} t}\right| j_{1} ;-t\right\rangle
$$

since the operators inserted in Eq. (9) are the identity operators. From Eq. (7), we have

$$
e^{-i H_{0} t}\left|j_{1} ;-t\right\rangle=e^{i E_{j_{1}} t} e^{-i H_{0} t}\left|j_{1} ; 0\right\rangle=\left|j_{1} ; 0\right\rangle
$$

and

$$
e^{i H_{0} t}\left|j_{2} ; t\right\rangle=e^{-i E_{j_{2}} t} e^{i H_{0} t}\left|j_{2} ; 0\right\rangle=\left|j_{2} ; 0\right\rangle
$$

Using these results and writing $e^{-i H 2 t}$ as $e^{-i H t} e^{-i H t}$ leads to

$$
S_{j_{2} j_{1}}=\left\langle j_{2} ; 0\left|\Omega_{-}^{\dagger} \Omega_{+}\right| j_{1} ; 0\right\rangle
$$

with

$$
\Omega_{+}=e^{-i H t} e^{i H_{0} t}
$$

and

$$
\Omega_{-}=e^{i H t} e^{-i H_{0} t}
$$

$\Omega_{+}$and $\Omega_{-}$are the Møller operators previously evoked. ${ }^{5,13}$ 


\subsection{Semiclassical propagators}

Introducing two closure relations in Eq. (13) between the states and the Møller operators, and two closure relations between the evolution operators of each Møller operator gives

$$
S_{j_{2} j_{1}}=\int d \bar{\phi}_{2} d \bar{\phi}_{1} d \phi_{2} d \phi_{1}\left\langle j_{2} ; 0 \mid \bar{\phi}_{2}\right\rangle\left\langle\bar{\phi}_{2}\left|e^{i H_{0} t}\right| \phi_{2}\right\rangle\left\langle\phi_{2}\left|e^{-i H 2 t}\right| \phi_{1}\right\rangle\left\langle\phi_{1}\left|e^{i H_{0} t}\right| \bar{\phi}_{1}\right\rangle\left\langle\bar{\phi}_{1} \mid j_{1} ; 0\right\rangle
$$

The addition of bars on top of the first and last integration variables in the propagation chain will make sense later. Using Eq. (6), this formula becomes

$$
S_{j_{2} j_{1}}=\frac{1}{2 \pi} \int d \bar{\phi}_{2} d \bar{\phi}_{1} d \phi_{2} d \phi_{1} e^{i\left(j_{1} \bar{\phi}_{1}-j_{2} \bar{\phi}_{2}\right)}\left\langle\bar{\phi}_{2}\left|e^{i H_{0} t}\right| \phi_{2}\right\rangle\left\langle\phi_{2}\left|e^{-i H 2 t}\right| \phi_{1}\right\rangle\left\langle\phi_{1}\left|e^{i H_{0} t}\right| \bar{\phi}_{1}\right\rangle
$$

We now wish to follow the traditional semiclassical approach which consists in replacing the three space-time propagators by their semiclassical limits, and integrating over the variables within the SPA. The first step requires the determination of these limits. Following Miller, ${ }^{1}$ we will be using the VVG propagator throughout this work.

For a one dimensional system of Cartesian coordinate $x$ and conjugate momentum $p$, this propagator $^{4}$ is:

$$
\left\langle x_{b}\left|e^{-i H t}\right| x_{a}\right\rangle=\sum_{k} \frac{e^{i\left(R_{k}\left(x_{b}, x_{a} ; t\right)-\frac{\pi}{2} \eta_{k}\right)}}{\left(\left.2 \pi i\left|\frac{\partial x_{b}}{\partial p_{a}}\right|_{x_{a}}\right|_{k}\right)^{1 / 2}} .
$$

The sum is over the set of classical trajectories connecting $\left(x_{a}, t_{a}\right)$ and $\left(x_{b}, t_{b}=t_{a}+t\right)$. If one takes $t$ as a parameter, both $p_{a}$ and $p_{b}$ are functions of $\left(x_{a}, x_{b}\right)$. In the following, this dependence will be formally stated by $p_{a} \equiv p_{a}\left(x_{a}, x_{b}\right)$ and $p_{b} \equiv p_{b}\left(x_{a}, x_{b}\right)$. However, the sum over $k$ suggests that these functions can be multivalued. $R_{k}\left(x_{b}, x_{a} ; t\right)$ is the action integral

$$
R\left(x_{b}, x_{a} ; t\right)=\int_{t_{a}}^{t_{b}=t_{a}+t} d \tau(p \dot{x}-H)
$$

along the $k^{t h}$ trajectory. $H$ is the classical function of Hamilton. $\eta_{k}$ is the Maslov index, i.e., the number of times $\left.\frac{\partial x}{\partial p_{a}}\right|_{x_{a}}$ takes the value 0 between $t_{a}$ and $t_{b}$. 
In order to apply Eq. (18) to our problem, we must take into account the fact that contrary to $x, \phi$ is a cyclic variable. $\phi$ and $\phi+2 k \pi$, where $k$ is any integer different from 0 , do indeed correspond to the same configuration. The semiclassical probability amplitude to go from $\left(\phi_{a}, t_{a}\right)$ to $\left(\phi_{b}, t_{b}\right)$, where both angles are restricted to the range $[0,2 \pi]$, will thus not only be constructed from the classical paths connecting $\left(\phi_{a}, t_{a}\right)$ and $\left(\phi_{b}, t_{b}\right)$ but also from those connecting $\left(\phi_{a}, t_{a}\right)$ and $\left(\phi_{b}+2 k \pi, t_{b}\right)$ with $k \neq 0$. Taking account of all the physically relevant paths is shown in Appendix A to ensure the exact propagation of the state of a free rotor. These considerations lead us to modify Eq. (17) as follows:

$$
\begin{array}{r}
S_{j_{2} j_{1}}=\frac{1}{2 \pi} \int d \bar{\phi}_{2} d \bar{\phi}_{1} d \phi_{2} d \phi_{1} e^{i\left(j_{1} \bar{\phi}_{1}-j_{2} \bar{\phi}_{2}\right)} \sum_{m, l, k}\left\langle\bar{\phi}_{2}+2 m \pi\left|e^{i H_{0} t}\right| \phi_{2}+2 l \pi\right\rangle \\
\left\langle\phi_{2}+2 l \pi\left|e^{-i H 2 t}\right| \phi_{1}+2 k \pi\right\rangle\left\langle\phi_{1}+2 k \pi\left|e^{i H_{0} t}\right| \bar{\phi}_{1}\right\rangle .
\end{array}
$$

The full propagation, thus, involves the infinite sum over segmented paths made of a first trajectory segment from $\bar{\phi}_{1}$ to $\phi_{1}+2 k \pi$, a second from $\phi_{1}+2 k \pi$ to $\phi_{2}+2 l \pi$ and a third from $\phi_{2}+2 l \pi$ to $\bar{\phi}_{2}+2 m \pi$. Now, integrating with respect to $\left(\bar{\phi}_{2}, \phi_{2}, \phi_{1}\right)$ over $[0,2 \pi]$ and summing over $m, l$ and $k$ simply amounts to integrating these variables over $[-\infty,+\infty]$ (see also Appendix A). $S_{j_{2} j_{1}}$ can thus be rewritten as

$$
S_{j_{2} j_{1}}=\frac{1}{2 \pi} \int_{-\infty}^{+\infty} d \bar{\phi}_{2} \int_{0}^{2 \pi} d \bar{\phi}_{1} e^{i\left(j_{1} \bar{\phi}_{1}-j_{2} \bar{\phi}_{2}\right)} Q\left(\bar{\phi}_{2}, \bar{\phi}_{1}\right)
$$

with

$$
Q\left(\bar{\phi}_{2}, \bar{\phi}_{1}\right)=\int_{-\infty}^{+\infty} d \phi_{2} \int_{-\infty}^{+\infty} d \phi_{1}\left\langle\bar{\phi}_{2}\left|e^{i H_{0} t}\right| \phi_{2}\right\rangle\left\langle\phi_{2}\left|e^{-i H 2 t}\right| \phi_{1}\right\rangle\left\langle\phi_{1}\left|e^{i H_{0} t}\right| \bar{\phi}_{1}\right\rangle
$$

We emphasize that Eqs. (20)-(22) are not quantum mechanically exact since apart from $\bar{\phi}_{1}$, the angles may take values outside the range $[0,2 \pi]$.

The expressions of $\left\langle\phi_{1}\left|e^{i H_{0} t}\right| \bar{\phi}_{1}\right\rangle$ and $\left\langle\bar{\phi}_{2}\left|e^{i H_{0} t}\right| \phi_{2}\right\rangle$ are deduced from Eq. (A.5) in Appendix 
A:

$$
\left\langle\phi_{1}\left|e^{i H_{0} t}\right| \bar{\phi}_{1}\right\rangle=\left(\frac{I}{-2 \pi i t}\right)^{1 / 2} e^{i \frac{I\left(\phi_{1}-\bar{\phi}_{1}\right)^{2}}{-2 t}}
$$

and

$$
\left\langle\bar{\phi}_{2}\left|e^{i H_{0} t}\right| \phi_{2}\right\rangle=\left(\frac{I}{-2 \pi i t}\right)^{1 / 2} e^{i \frac{I\left(\bar{\phi}_{2}-\phi_{2}\right)^{2}}{-2 t}} .
$$

The expression of $\left\langle\phi_{2}\left|e^{-i H 2 t}\right| \phi_{1}\right\rangle$ is straightforwardly deduced from Eqs. (18) and (19):

$$
\left\langle\phi_{2}\left|e^{-i H 2 t}\right| \phi_{1}\right\rangle=\sum_{k} \frac{e^{i\left(R_{k}\left(\phi_{2}, \phi_{1} ; 2 t\right)-\frac{\pi}{2} \eta_{k}\right)}}{\left(\left.2 \pi i\left|\frac{\partial \phi_{2}}{\partial J_{1}}\right|_{\phi_{1}}\right|_{k}\right)^{1 / 2}}
$$

with

$$
R_{k}\left(\phi_{2}, \phi_{1} ; 2 t\right)=\int_{-t}^{t} d \tau(J \dot{\phi}-H)=\int_{\phi_{1}}^{\phi_{2}} J d \phi-\int_{-t}^{t} d \tau H
$$

The various terms have been previously defined. Here,

$$
H=\frac{J^{2}}{2 I}+V(\phi, \tau)
$$

An example of three-segment path contributing to $S_{j_{2} j_{1}}$ is drawn in Fig. 1 . The blue segment, run backward in time from $\left(\bar{\phi}_{1}, 0\right)$ to $\left(\phi_{1},-t\right)$, is used to calculate the right-handside (RHS) of Eq. (23). The green segment, run forward in time from $\left(\phi_{1},-t\right)$ to $\left(\phi_{2}, t\right)$, is used to calculate the RHS of Eq. (25). Finally, the red segment, run backward in time from $\left(\phi_{2}, t\right)$ to $\left(\bar{\phi}_{2}, 0\right)$, is used to calculate the RHS of Eq. (24). The backward propagations being performed by means of the free Hamiltonian, the blue and red segments are necessarily linear. The green segment involves linear parts for asymptotically large values of $|\tau|$ where $V(\phi, \tau)$ is zero. 


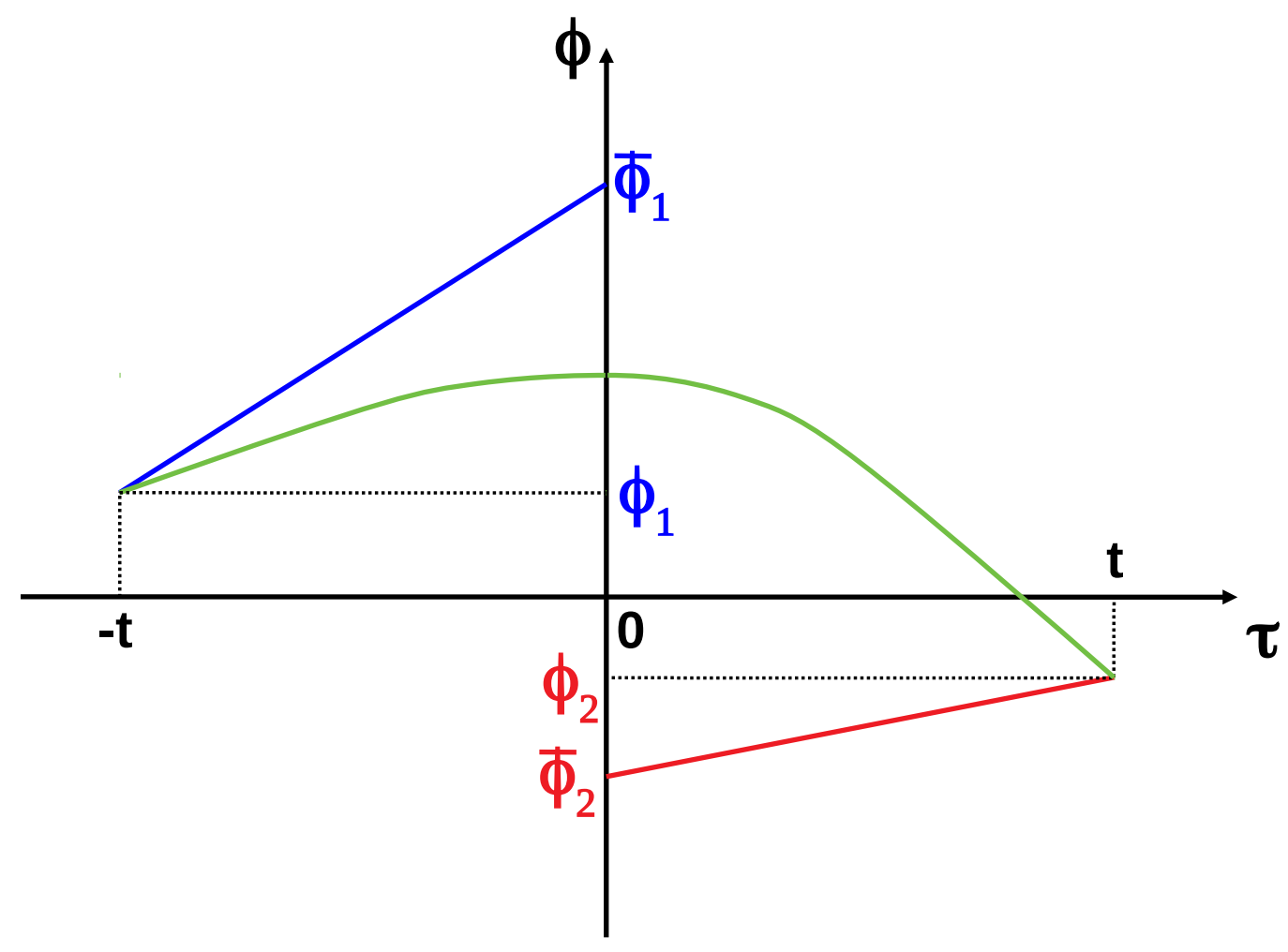

Figure 1: Example of three-segment path used to calculate the semiclassical propagators given by Eqs. (23)-(25).

\subsection{Shifted angles as a consequence of the stationary phase ap- proximation}

We are now in a position to integrate the RHS of Eq. (22) with respect to $\phi_{1}$ and $\phi_{2}$ within the SPA. ${ }^{4}$ In two dimensions, this approximation results in

$$
\int d \phi_{2} d \phi_{1} g\left(\phi_{2}, \phi_{1}\right) e^{i f\left(\phi_{2}, \phi_{1}\right)} \approx \sum_{l} g\left(\phi_{2 l}, \phi_{1 l}\right) e^{i\left[f\left(\phi_{2 l}, \phi_{1 l}\right)-\pi \nu_{l} / 2\right]} \frac{2 \pi i}{\left|\Delta_{l}\right|^{1 / 2}}
$$


where $\left(\phi_{2 l}, \phi_{1 l}\right)$ is the $l^{t h}$ stationnary point of $f, \Delta_{l}$ is the discriminant of the Hessian of $f$, given by

$$
\Delta_{l}=\left.\left.\frac{\partial^{2} f}{\partial \phi_{1}^{2}}\right|_{l} \frac{\partial^{2} f}{\partial \phi_{2}^{2}}\right|_{l}-\left(\left.\frac{\partial^{2} f}{\partial \phi_{2} \partial \phi_{1}}\right|_{l}\right)^{2}
$$

and $\nu_{l}$ is the number of negative eigenvalues of the Hessian ( $g$ is assumed to evolve smoothly enough around the stationary points). In order to alleviate notation, we momentarily ignore the sum over $k$ and $l$ in Eqs. (25) and (28) as well as the phase indices $\eta_{k}$ and $\nu_{l}$. This sum will be considered at the end of Sec. 2.5 and the phase indices in Secs. 3 and 4. Identifying the left-hand-side of Eq. (28) with the RHS of Eq. (22) and using Eqs. (23)-(25) allows to express $Q\left(\bar{\phi}_{2}, \bar{\phi}_{1}\right)$ as the RHS of Eq. (28) with

$$
g\left(\phi_{2}, \phi_{1}\right)=\left(\frac{I}{2 \pi i t}\right) \frac{1}{\left(2 \pi i\left|\frac{\partial \phi_{2}}{\partial J_{1}}\right|_{\phi_{1}} \mid\right)^{1 / 2}}
$$

and

$$
f\left(\phi_{2}, \phi_{1}\right)=R\left(\phi_{2}, \phi_{1} ; 2 t\right)-\frac{I}{2 t}\left[\left(\phi_{1}-\bar{\phi}_{1}\right)^{2}+\left(\bar{\phi}_{2}-\phi_{2}\right)^{2}\right]
$$

$f$ is thus stationary for

$$
\left.\frac{\partial f}{\partial \phi_{1}}\right|_{\phi_{2}}=\left.\frac{\partial R}{\partial \phi_{1}}\right|_{\phi_{2}}-\frac{I}{t}\left(\phi_{1}-\bar{\phi}_{1}\right)=0
$$

and

$$
\left.\frac{\partial f}{\partial \phi_{2}}\right|_{\phi_{1}}=\left.\frac{\partial R}{\partial \phi_{2}}\right|_{\phi_{1}}+\frac{I}{t}\left(\bar{\phi}_{2}-\phi_{2}\right)=0 .
$$

Moreover,

$$
\left.\frac{\partial R}{\partial \phi_{1}}\right|_{\phi_{2}}=-J_{1}
$$

and

$$
\left.\frac{\partial R}{\partial \phi_{2}}\right|_{\phi_{1}}=J_{2}
$$


(these two canonical equations of classical mechanics are deduced from the RHS of Eq. (26)). We thus arrive at

$$
\phi_{1}=\bar{\phi}_{1}-\frac{t}{I} J_{1}
$$

and

$$
\phi_{2}=\bar{\phi}_{2}+\frac{t}{I} J_{2}
$$

Since $J_{1} / I$ and $J_{2} / I$ are angular frequencies, Eqs. (36) and (37) are recognized to be Miller's equations (2) and (3). $\phi_{1}$ and $\phi_{2}$ are thus the natural angles of the problem, whereas $\bar{\phi}_{1}$ and $\bar{\phi}_{2}$ are Miller's shifted angles, justifying a posteriori the assignment of bars to these variables in Eq. (16).

The physics underlying Eqs. (36) and (37) is schematically represented in Fig. 2. The trajectory going from $\left(-t, \phi_{1}\right)$ to $\left(t, \phi_{2}\right)$ is now represented by the orange curve for the negative times, and the green curve for the positive ones. Before $-t$ and after $t$, the interaction potential is 0 and the angular momentum $J=I \dot{\phi}$ is a constant of motion equal to $J_{1}$ and $J_{2}$, respectively. The slopes of the orange and green curves at $\left(-t, \phi_{1}\right)$ and $\left(t, \phi_{2}\right)$ are thus given by $J_{1} / I$ and $J_{2} / I$. But Eqs. (36) and (37), resulting from the stationary phase approximation, force the slopes $\left(\bar{\phi}_{1}-\phi_{1}\right) / t$ and $\left(\phi_{2}-\bar{\phi}_{2}\right) / t$ of the blue and red straight lines to be also given by $J_{1} / I$ and $J_{2} / I$. In other words, at the segment contact points, the momenta along the two segments are equal, i.e., the orange curve and the blue straight line are superimposed at $-t$ (and before) while the green curve and the red straight line are superimposed at $t$ (and after). The classical analog of the action of $\Omega_{+}$is the run of the blue straight line backward in time during $t$ followed by the run of the orange line forward in time during $t$. Besides, the classical analog of the action of $\Omega_{-}$is the run of the red straight line forward in time during $t$ followed by the run of the green line backward in time during $t$. In actual calculations of Miller's type, however, one performs the run of the blue straight line backward in time during $t$, then the run of the orange and green lines forward in time during $2 t$ and finally the run of the red straight line backward in time during $t$. 


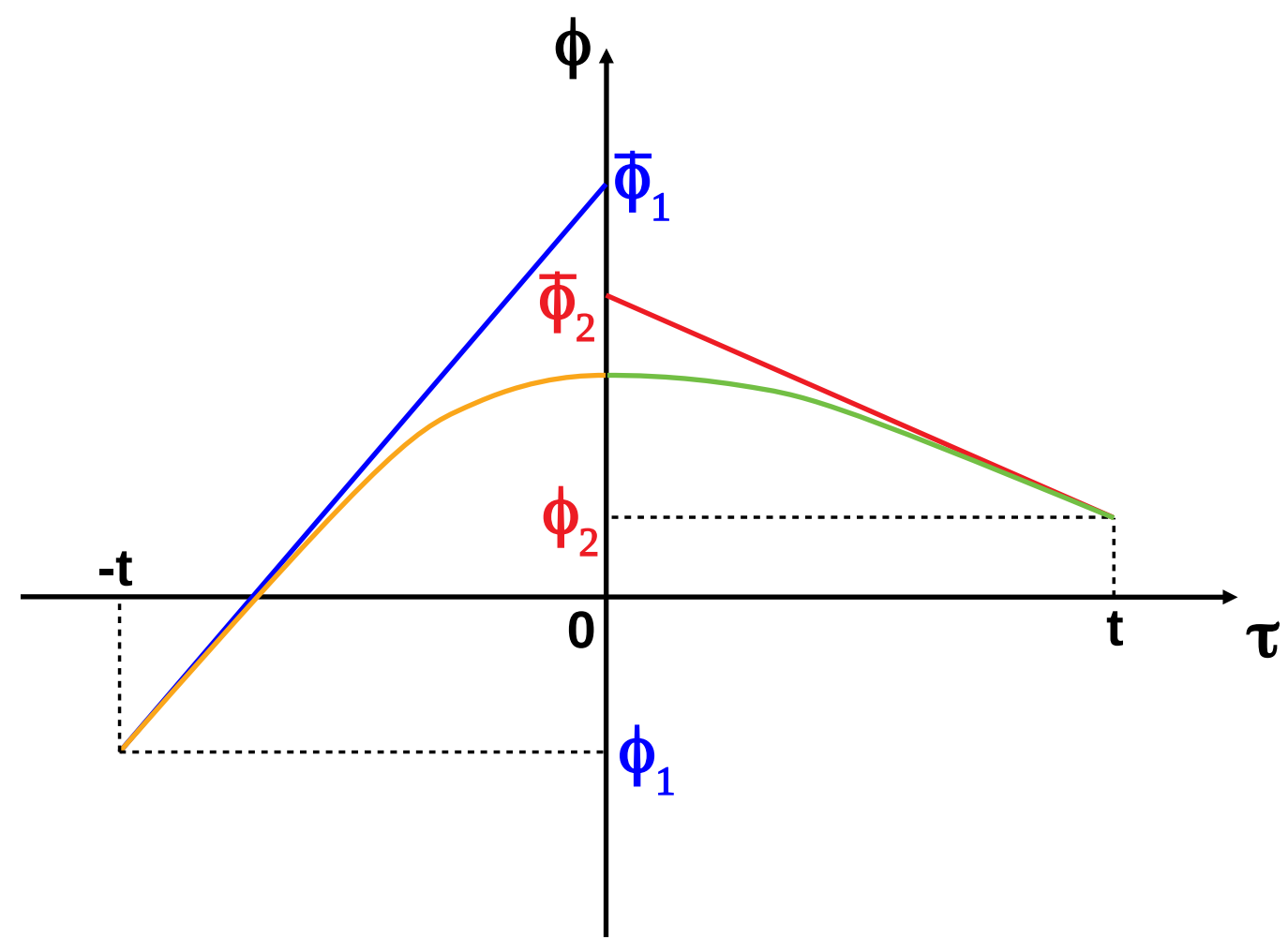

Figure 2: Schematic representation of the physics underlying Eqs. (36) and (37). Among the infinite and continuous set of three-segment paths represented in Fig. 1, the stationary phase approximation picks the discrete set of those for which the angles between segments are zero at $-t$ and $t$. The momenta along the segments at these instants are thus equal (see text for more details).

The second derivatives in Eq. (29) are found from Eqs. (32)-(35) to be given by

$$
\begin{gathered}
\frac{\partial^{2} f}{\partial \phi_{1}^{2}}=-\left.\frac{\partial J_{1}}{\partial \phi_{1}}\right|_{\phi_{2}}-\frac{I}{t} \\
\frac{\partial^{2} f}{\partial \phi_{2}^{2}}=\left.\frac{\partial J_{2}}{\partial \phi_{2}}\right|_{\phi_{1}}-\frac{I}{t}
\end{gathered}
$$

and

$$
\frac{\partial^{2} f}{\partial \phi_{2} \partial \phi_{1}}=-\left.\frac{\partial J_{1}}{\partial \phi_{2}}\right|_{\phi_{1}}
$$


We have everything to derive the expression of $Q\left(\bar{\phi}_{2}, \bar{\phi}_{1}\right)$. The prerequisite, however, is to make the natural angles $\phi_{2}$ and $\phi_{1}$ disappear from all the partial derivatives involved in the expression of $Q\left(\bar{\phi}_{2}, \bar{\phi}_{1}\right)$. In the section below, we play with these derivatives and use Eqs. (36) and (37) so as to satisfy this requirement.

\subsection{Juggling with partial derivatives to make natural angles dis- appear}

Six variables have appeared in the developments: $\phi_{1}, \phi_{2}, J_{1}, J_{2}, \bar{\phi}_{1}$ and $\bar{\phi}_{2}$. Moreover, we have four relations available between them: Eq. (36), Eq. (37), $J_{1} \equiv J_{1}\left(\phi_{1}, \phi_{2}\right)$ and $J_{2} \equiv J_{2}\left(\phi_{1}, \phi_{2}\right)$ (the two last relations are deduced from the comments following Eq. (18)). Therefore, only two variables are independent. Each variable can thus be seen as a function of two variables among the five remaining ones (obviously, one has to carefully deal with branch issues). We will repeatedly use this property in the following.

Neither $\left.\frac{\partial J_{1}}{\partial \phi_{1}}\right|_{\phi_{2}}$ nor $\left.\frac{\partial J_{2}}{\partial \phi_{2}}\right|_{\phi_{1}}$ (see Eqs. (38) and (39)) are intuitively appealing or convenient for actual calculations. On the contrary, the derivative of a final variable in terms of an initial variable with the remaining initial variable held constant, like $\left.\frac{\partial \phi_{2}}{\partial J_{1}}\right|_{\phi_{1}}$, are obvious to conceive and compute. We now express the former in terms of the latter, starting with $\left.\frac{\partial J_{1}}{\partial \phi_{1}}\right|_{\phi_{2}}$. As we have just seen, we can write $\phi_{2} \equiv \phi_{2}\left(\phi_{1}, J_{1}\right)$ and deduce the differential

$$
d \phi_{2}=\left.\frac{\partial \phi_{2}}{\partial \phi_{1}}\right|_{J_{1}} d \phi_{1}+\left.\frac{\partial \phi_{2}}{\partial J_{1}}\right|_{\phi_{1}} d J_{1}
$$

Now, looking for the $\phi_{1}$ dependence of $J_{1}$ with $\phi_{2}$ held constant amounts to take $d \phi_{2}$ at 0 in the above equation. The ratio $d J_{1} / d \phi_{1}$ obtained by doing so is just the expression of $\left.\frac{\partial J_{1}}{\partial \phi_{1}}\right|_{\phi_{2}}$ we sought:

$$
\left.\frac{\partial J_{1}}{\partial \phi_{1}}\right|_{\phi_{2}}=-\left.\frac{\partial \phi_{2}}{\partial \phi_{1}}\right|_{J_{1}} /\left.\frac{\partial \phi_{2}}{\partial J_{1}}\right|_{\phi_{1}} .
$$

The same reasoning switching labels 1 and 2 would not lead to a satisfying expression of $\left.\frac{\partial J_{2}}{\partial \phi_{2}}\right|_{\phi_{1}}$, for the variables held constant in the partial derivatives would be $J_{2}$ and $\phi_{2}$ rather 
than $J_{1}$ and $\phi_{1}$. Instead, we use the fact that $J_{2} \equiv J_{2}\left(\phi_{1}, J_{1}\right)$ to obtain

$$
d J_{2}=\left.\frac{\partial J_{2}}{\partial \phi_{1}}\right|_{J_{1}} d \phi_{1}+\left.\frac{\partial J_{2}}{\partial J_{1}}\right|_{\phi_{1}} d J_{1} .
$$

Now, looking for the $\phi_{2}$ dependence of $J_{2}$ with $\phi_{1}$ held constant amounts to take $d \phi_{1}$ at 0 in the above equation as well as in Eq. (41). The ratio $d J_{2} / d \phi_{2}$ obtained by doing so is the desired expression of $\left.\frac{\partial J_{2}}{\partial \phi_{2}}\right|_{\phi_{1}}$ :

$$
\left.\frac{\partial J_{2}}{\partial \phi_{2}}\right|_{\phi_{1}}=\left.\frac{\partial J_{2}}{\partial J_{1}}\right|_{\phi_{1}} /\left.\frac{\partial \phi_{2}}{\partial J_{1}}\right|_{\phi_{1}} .
$$

We note that $\left.\frac{\partial J_{1}}{\partial \phi_{1}}\right|_{\phi_{2}}$ and $\left.\frac{\partial J_{2}}{\partial \phi_{2}}\right|_{\phi_{1}}$ depend on the three partial derivatives $\left.\frac{\partial \phi_{2}}{\partial \phi_{1}}\right|_{J_{1}},\left.\frac{\partial \phi_{2}}{\partial J_{1}}\right|_{\phi_{1}}$ and $\left.\frac{\partial J_{2}}{\partial J_{1}}\right|_{\phi_{1}}$ (see Eqs. (42) and (44)). As a consequence, the developments going from Eq. (28) to Eq. (40) show that the pre-exponential factor of the integrand of $Q\left(\bar{\phi}_{2}, \bar{\phi}_{1}\right)$ depends solely on these three derivatives.

We now use Eqs. (36) and (37) to make $\left(\bar{\phi}_{2}, \bar{\phi}_{1}\right)$ appear in, and $\left(\phi_{2}, \phi_{1}\right)$ disappear from these derivatives. Eq. (36) shows that deriving with respect to $\phi_{1}$ with $J_{1}$ held constant is equivalent to deriving with respect to $\bar{\phi}_{1}$ with $J_{1}$ held constant. Using Eq. (37), we thus get

$$
\left.\frac{\partial \phi_{2}}{\partial \phi_{1}}\right|_{J_{1}}=\left.\frac{\partial \bar{\phi}_{2}}{\partial \bar{\phi}_{1}}\right|_{J_{1}}+\left.\frac{t}{I} \frac{\partial J_{2}}{\partial \bar{\phi}_{1}}\right|_{J_{1}} .
$$

For the partial derivatives with respect to $J_{1}$ with $\phi_{1}$ held constant, we deduce from $\phi_{2} \equiv$ $\phi_{2}\left(\bar{\phi}_{1}, J_{1}\right)$ the differential

$$
d \phi_{2}=\left.\frac{\partial \phi_{2}}{\partial \bar{\phi}_{1}}\right|_{J_{1}} d \bar{\phi}_{1}+\left.\frac{\partial \phi_{2}}{\partial J_{1}}\right|_{\bar{\phi}_{1}} d J_{1}
$$

Moreover, Eq. (36) implies

$$
d \phi_{1}=d \bar{\phi}_{1}-\frac{t}{I} d J_{1}
$$

Looking for the variation of $\phi_{2}$ in terms of $J_{1}$ with $\phi_{1}$ held constant, we proceed as previously: $d \phi_{1}$ is taken at 0 in the above identity, thus allowing to replace $d \bar{\phi}_{1}$ by $t d J_{1} / I$ in Eq. (46). 
One then obtains

$$
\left.\frac{\partial \phi_{2}}{\partial J_{1}}\right|_{\phi_{1}}=\left.\frac{\partial \phi_{2}}{\partial J_{1}}\right|_{\bar{\phi}_{1}}+\left.\frac{t}{I} \frac{\partial \phi_{2}}{\partial \bar{\phi}_{1}}\right|_{J_{1}}
$$

Replacing $\phi_{2}$ in the RHS of this equation by the RHS of Eq. (37) leads to

$$
\left.\frac{\partial \phi_{2}}{\partial J_{1}}\right|_{\phi_{1}}=\left.\frac{\partial \bar{\phi}_{2}}{\partial J_{1}}\right|_{\bar{\phi}_{1}}+\frac{t}{I}\left(\left.\frac{\partial \bar{\phi}_{2}}{\partial \bar{\phi}_{1}}\right|_{J_{1}}+\left.\frac{\partial J_{2}}{\partial J_{1}}\right|_{\bar{\phi}_{1}}\right)+\left.\left(\frac{t}{I}\right)^{2} \frac{\partial J_{2}}{\partial \bar{\phi}_{1}}\right|_{J_{1}} .
$$

For the last derivative, $\left.\frac{\partial J_{2}}{\partial J_{1}}\right|_{\phi_{1}}$, one deduces from $J_{2} \equiv J_{2}\left(\bar{\phi}_{1}, J_{1}\right)$ the differential

$$
d J_{2}=\left.\frac{\partial J_{2}}{\partial \bar{\phi}_{1}}\right|_{J_{1}} d \bar{\phi}_{1}+\left.\frac{\partial J_{2}}{\partial J_{1}}\right|_{\bar{\phi}_{1}} d J_{1}
$$

Repeating the same reasoning as for $\left.\frac{\partial \phi_{2}}{\partial J_{1}}\right|_{\phi_{1}}$, we arrive at

$$
\left.\frac{\partial J_{2}}{\partial J_{1}}\right|_{\phi_{1}}=\left.\frac{\partial J_{2}}{\partial J_{1}}\right|_{\bar{\phi}_{1}}+\left.\frac{t}{I} \frac{\partial J_{2}}{\partial \bar{\phi}_{1}}\right|_{J_{1}}
$$

$\phi_{2}$ and $\phi_{1}$ have thus disappeared from all the partial derivatives involved in the expression of $Q\left(\bar{\phi}_{2}, \bar{\phi}_{1}\right)$.

\subsection{Recovering Miller's original SC-IVR expression}

We now perform the last steps of algebra that allow to recover the original SC-IVR expression of $S$ matrix elements. We start with the Hessian $\Delta$ given by Eqs. (29) and Eqs. (38)-(40). From Eqs. (38), (42) and (45), we have

$$
\frac{\partial^{2} f}{\partial \phi_{1}^{2}}=\left(\left.\frac{\partial \bar{\phi}_{2}}{\partial \bar{\phi}_{1}}\right|_{J_{1}}+\left.\frac{t}{I} \frac{\partial J_{2}}{\partial \bar{\phi}_{1}}\right|_{J_{1}}-\left.\frac{I}{t} \frac{\partial \phi_{2}}{\partial J_{1}}\right|_{\phi_{1}}\right) /\left.\frac{\partial \phi_{2}}{\partial J_{1}}\right|_{\phi_{1}}
$$

Replacing $\left.\frac{\partial \phi_{2}}{\partial J_{1}}\right|_{\phi_{1}}$ in the numerator of the above expression by the RHS of Eq. (49) leads to

$$
\frac{\partial^{2} f}{\partial \phi_{1}^{2}}=-\left(\left.\frac{\partial J_{2}}{\partial J_{1}}\right|_{\bar{\phi}_{1}}+\left.\frac{I}{t} \frac{\partial \bar{\phi}_{2}}{\partial J_{1}}\right|_{\bar{\phi}_{1}}\right) /\left.\frac{\partial \phi_{2}}{\partial J_{1}}\right|_{\phi_{1}}
$$


In the same way, Eqs. (39), (44) and (51) give

$$
\frac{\partial^{2} f}{\partial \phi_{2}^{2}}=\left(\left.\frac{\partial J_{2}}{\partial J_{1}}\right|_{\bar{\phi}_{1}}+\left.\frac{t}{I} \frac{\partial J_{2}}{\partial \bar{\phi}_{1}}\right|_{J_{1}}-\left.\frac{I}{t} \frac{\partial \phi_{2}}{\partial J_{1}}\right|_{\phi_{1}}\right) /\left.\frac{\partial \phi_{2}}{\partial J_{1}}\right|_{\phi_{1}}
$$

and substituting the RHS of Eq. (49) to $\left.\frac{\partial \phi_{2}}{\partial J_{1}}\right|_{\phi_{1}}$ in the numerator leads to

$$
\frac{\partial^{2} f}{\partial \phi_{1}^{2}}=-\left(\left.\frac{\partial \bar{\phi}_{2}}{\partial \bar{\phi}_{1}}\right|_{\bar{\phi}_{1}}+\left.\frac{I}{t} \frac{\partial \bar{\phi}_{2}}{\partial J_{1}}\right|_{\bar{\phi}_{1}}\right) /\left.\frac{\partial \phi_{2}}{\partial J_{1}}\right|_{\phi_{1}} .
$$

Hence, $\Delta$ (see Eqs. (29)) is given by

$$
\Delta=\left[\left(\left.\frac{\partial \bar{\phi}_{2}}{\partial \bar{\phi}_{1}}\right|_{\bar{\phi}_{1}}+\left.\frac{I}{t} \frac{\partial \bar{\phi}_{2}}{\partial J_{1}}\right|_{\bar{\phi}_{1}}\right)\left(\left.\frac{\partial J_{2}}{\partial J_{1}}\right|_{\bar{\phi}_{1}}+\left.\frac{I}{t} \frac{\partial \bar{\phi}_{2}}{\partial J_{1}}\right|_{\bar{\phi}_{1}}\right)-1\right] /\left(\left.\frac{\partial \phi_{2}}{\partial J_{1}}\right|_{\phi_{1}}\right)^{2} .
$$

The last step makes use of Liouville theorem, ${ }^{19}$

$$
d \bar{\phi}_{2} d J_{2}=d \bar{\phi}_{1} d J_{1}
$$

(this theorem being valid for the initial backward run from 0 to $-t$, the forward run from $-t$ to $t$ and the final backward run from $t$ to 0 , it is valid for the whole run). Now, independently on Liouville theorem,

$$
d \bar{\phi}_{2} d J_{2}=\left|\begin{array}{ll}
\left.\frac{\partial \bar{\phi}_{2}}{\partial \bar{\phi}_{1}}\right|_{J_{1}} & \left.\frac{\partial \bar{\phi}_{2}}{\partial J_{1}}\right|_{\bar{\phi}_{1}} \\
\left.\frac{\partial J_{2}}{\partial \bar{\phi}_{1}}\right|_{J_{1}} & \left.\frac{\partial J_{2}}{\partial J_{1}}\right|_{\bar{\phi}_{1}}
\end{array}\right| d \bar{\phi}_{1} d J_{1}
$$

Consequently,

$$
1=\left.\left.\frac{\partial \bar{\phi}_{2}}{\partial \bar{\phi}_{1}}\right|_{J_{1}} \frac{\partial J_{2}}{\partial J_{1}}\right|_{\bar{\phi}_{1}}-\left.\left.\frac{\partial \bar{\phi}_{2}}{\partial J_{1}}\right|_{\bar{\phi}_{1}} \frac{\partial J_{2}}{\partial \bar{\phi}_{1}}\right|_{J_{1}} .
$$

Replacing 1 in Eq. (56) by the RHS of Eq. (59) leads to

$$
\Delta=\left.\frac{\partial \bar{\phi}_{2}}{\partial J_{1}}\right|_{\bar{\phi}_{1}}\left[\left.\left(\frac{I}{t}\right)^{2} \frac{\partial \bar{\phi}_{2}}{\partial J_{1}}\right|_{\bar{\phi}_{1}}+\frac{I}{t}\left(\left.\frac{\partial \bar{\phi}_{2}}{\partial \bar{\phi}_{1}}\right|_{J_{1}}+\left.\frac{\partial J_{2}}{\partial J_{1}}\right|_{\bar{\phi}_{1}}\right)+\left.\frac{\partial J_{2}}{\partial \bar{\phi}_{1}}\right|_{J_{1}}\right] /\left(\left.\frac{\partial \phi_{2}}{\partial J_{1}}\right|_{\phi_{1}}\right)^{2} .
$$


The term within the square bracket is recognized from Eq. (49) to be equal to $\left.\left(\frac{I}{t}\right)^{2} \frac{\partial \phi_{2}}{\partial J_{1}}\right|_{\phi_{1}}$ so we finally obtain

$$
\Delta=\left.\left(\frac{I}{t}\right)^{2} \frac{\partial \bar{\phi}_{2}}{\partial J_{1}}\right|_{\bar{\phi}_{1}} /\left.\frac{\partial \phi_{2}}{\partial J_{1}}\right|_{\phi_{1}}
$$

Now, we recall that $Q\left(\bar{\phi}_{2}, \bar{\phi}_{1}\right)$ is given by the RHS of Eq. (28) with $g\left(\phi_{2}, \phi_{1}\right)$ given by Eq. (30), $f\left(\phi_{2}, \phi_{1}\right)$ given by Eq. (31), and $\Delta$ given above. Therefore, using Eqs. (36) and (37), we arrive at

$$
Q\left(\bar{\phi}_{2}, \bar{\phi}_{1}\right)=\left.\frac{1}{(2 \pi i)^{1 / 2}}\left|\frac{\partial \bar{\phi}_{2}}{\partial J_{1}}\right|_{\bar{\phi}_{1}}\right|^{-1 / 2} \exp \left[i\left(R\left(\phi_{2}, \phi_{1} ; 2 t\right)-\left(\frac{J_{2}^{2}+J_{1}^{2}}{2 I}\right) t\right)\right]
$$

Applying the integration by part formula to Eq. (26) allows to rewrite $R\left(\phi_{2}, \phi_{1} ; 2 t\right)$ as

$$
R\left(\phi_{2}, \phi_{1} ; 2 t\right)=J_{2} \phi_{2}-J_{1} \phi_{1}-\int_{-t}^{t} d \tau(\phi \dot{J}+H)
$$

Using this expression in Eq. (62) and replacing $\phi_{2}$ and $\phi_{1}$ by the RHS of Eqs. (36) and (37) leads to

$$
Q\left(\bar{\phi}_{2}, \bar{\phi}_{1}\right)=\left.\frac{1}{(2 \pi i)^{1 / 2}}\left|\frac{\partial \bar{\phi}_{2}}{\partial J_{1}}\right|_{\bar{\phi}_{1}}\right|^{-1 / 2} e^{i\left(J_{2} \bar{\phi}_{2}-J_{1} \bar{\phi}_{1}+\bar{\Theta}\right)}
$$

with

$$
\bar{\Theta}=\left(\frac{J_{2}^{2}+J_{1}^{2}}{2 I}\right) t-\int_{-t}^{t} d \tau(\phi \dot{J}+H)=\left(\frac{J_{2}^{2}+J_{1}^{2}}{2 I}\right) t-\int_{J_{1}}^{J_{2}} \phi d J-\int_{-t}^{t} d \tau H
$$

From Eqs. (21) and (64), we get

$S_{j_{2} j_{1}}=\left.\frac{1}{2 \pi(2 \pi i)^{1 / 2}} \int_{-\infty}^{+\infty} d \bar{\phi}_{2} \int_{0}^{2 \pi} d \bar{\phi}_{1} \sum_{\text {Paths }}\left|\frac{\partial \bar{\phi}_{2}}{\partial J_{1}}\right|_{\bar{\phi}_{1}}\right|^{-1 / 2} \exp \left\{i\left(\left[j_{1}-J_{1}\right] \bar{\phi}_{1}+\left[J_{2}-j_{2}\right] \bar{\phi}_{2}+\bar{\Theta}\right)\right\}$.

Let us recall that for simplity's sake, we had ignored the sum over $k$ and $l$ in Eqs. (25) and (28) (see a few lines below Eq. (29)). Eq. (66) now takes this sum into account via the 
$\sum_{\text {Paths }}$ term. Since $\bar{\phi}_{2} \equiv \bar{\phi}_{2}\left(\bar{\phi}_{1}, J_{1}\right)$, this sum is over those values of $J_{1}$ leading from $\bar{\phi}_{1}$ to the value $\bar{\phi}_{2}$ of $\bar{\phi}_{2}\left(\bar{\phi}_{1}, J_{1}\right)$. The discrete set of paths thus defined evolve when varying $\bar{\phi}_{1}$ and $\bar{\phi}_{2}$.

Following Miller, ${ }^{14}$ we integrate the RHS of this equation over $\bar{\phi}_{1}$ within the SPA, i.e., by means of Eq. (A.11). Calling $\varphi$ the phase of the integrand, we have

$$
\left.\frac{\partial \varphi}{\partial \bar{\phi}_{1}}\right|_{\bar{\phi}_{2}}=j_{1}-J_{1}-\left.\bar{\phi}_{1} \frac{\partial J_{1}}{\partial \bar{\phi}_{1}}\right|_{\bar{\phi}_{2}}+\left.\bar{\phi}_{2} \frac{\partial J_{2}}{\partial \bar{\phi}_{1}}\right|_{\bar{\phi}_{2}}+\left.\frac{\partial \bar{\Theta}}{\partial \bar{\phi}_{1}}\right|_{\bar{\phi}_{2}}
$$

with from the RHS of Eq. (65),

$$
\left.\frac{\partial \bar{\Theta}}{\partial \bar{\phi}_{1}}\right|_{\bar{\phi}_{2}}=\left.\frac{J_{2}}{I} t \frac{\partial J_{2}}{\partial \bar{\phi}_{1}}\right|_{\bar{\phi}_{2}}+\left.\frac{J_{1}}{I} t \frac{\partial J_{1}}{\partial \bar{\phi}_{1}}\right|_{\bar{\phi}_{2}}-\left.\phi_{2} \frac{\partial J_{2}}{\partial \bar{\phi}_{1}}\right|_{\bar{\phi}_{2}}+\left.\phi_{1} \frac{\partial J_{1}}{\partial \bar{\phi}_{1}}\right|_{\bar{\phi}_{2}} .
$$

Using Eqs. (36) and (37), Eqs. (67) and (68) simplify to

$$
\left.\frac{\partial \varphi}{\partial \bar{\phi}_{1}}\right|_{\bar{\phi}_{2}}=j_{1}-J_{1}
$$

Hence, the values of $\bar{\phi}_{1}$ making $\varphi$ stationary satisfy $J_{1}\left(\bar{\phi}_{2}, \bar{\phi}_{1}\right)=j_{1}$. Moreover,

$$
\frac{\partial^{2} \varphi}{\partial \bar{\phi}_{1}^{2}}=-\left.\frac{\partial J_{1}}{\partial \bar{\phi}_{1}}\right|_{\bar{\phi}_{2}}=\left.\frac{\partial \bar{\phi}_{2}}{\partial \bar{\phi}_{1}}\right|_{J_{1}} /\left.\frac{\partial \bar{\phi}_{2}}{\partial J_{1}}\right|_{\bar{\phi}_{1}}
$$

The second equality is obtained in the same way as Eq. (42). Using Eqs. (A.11), (70) and the fact that $j_{1}=J_{1}$, Eq. (66) becomes

$$
S_{j_{2} j_{1}}=\left.\frac{1}{2 \pi} \int_{-\infty}^{+\infty} d \bar{\phi}_{2} \sum_{\text {Branches }}\left|\frac{\partial \bar{\phi}_{2}}{\partial \bar{\phi}_{1}}\right|_{J_{1}}\right|^{-1 / 2} \exp \left\{i\left(\left[J_{2}-j_{2}\right] \bar{\phi}_{2}+\bar{\Theta}\right)\right\}
$$

where $\bar{\Theta}$ is given by Eqs. (65) with $J_{1}=j_{1}$. The sum is over the possible branches of the function $\bar{\phi}_{1} \equiv \bar{\phi}_{1}\left(\bar{\phi}_{2}, j_{1}\right)$, obtained by inverting $\bar{\phi}_{2} \equiv \bar{\phi}_{2}\left(\bar{\phi}_{1}, j_{1}\right)$. A hypothetical representation of these functions is given in Fig. 3 for the case of three branches. While $\bar{\phi}_{2} \equiv \bar{\phi}_{2}\left(\bar{\phi}_{1}, j_{1}\right)$ is 
obviously single-valued, $\bar{\phi}_{1} \equiv \bar{\phi}_{1}\left(\bar{\phi}_{2}, j_{1}\right)$ is multi-valued. The idea is now to make the change

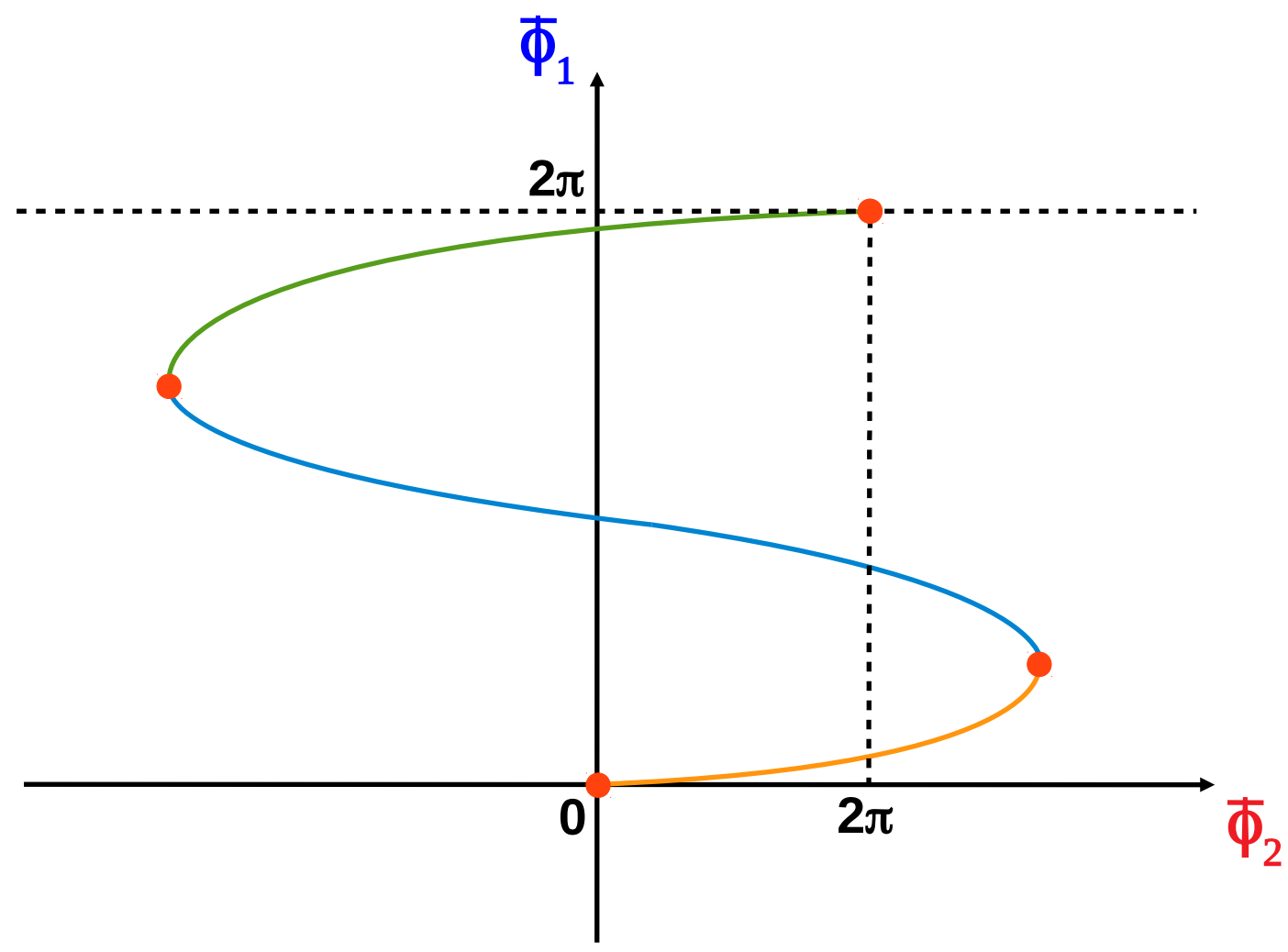

Figure 3: Representation of the function $\bar{\phi}_{2} \equiv \bar{\phi}_{2}\left(\bar{\phi}_{1}, j_{1}\right)$ and its inverse $\bar{\phi}_{1} \equiv \bar{\phi}_{1}\left(\bar{\phi}_{2}, j_{1}\right)$ in the hypothetical situation where the inverse function involves three branches (of different colors and delimited by dots). Note that while $\bar{\phi}_{1}$ belongs to the range $[0,2 \pi], \bar{\phi}_{2}$ is unbound in principle; this is particularly the case for chaotic dynamics involving long residence times within the interaction region. For $\bar{\phi}_{1}=2 \pi, \bar{\phi}_{2}$ is $2 \pi$ larger than its value at $\bar{\phi}_{1}=0$ owing to the periodicity of the problem.

of variable $d \bar{\phi}_{2}=\left|\frac{\partial \bar{\phi}_{2}}{\partial \bar{\phi}_{1}}\right|_{J_{1}} \mid d \bar{\phi}_{1}$ for each branch. ${ }^{14}$ This makes the sum over branches disappear and leads, ultimately, to the expected Eq. (29) of Ref. ${ }^{14}$

$$
S_{j_{2} j_{1}}=\left.\frac{1}{2 \pi} \int_{0}^{2 \pi} d \bar{\phi}_{1}\left|\frac{\partial \bar{\phi}_{2}}{\partial \bar{\phi}_{1}}\right|_{J_{1}}\right|^{1 / 2} \exp \left\{i\left(\left[J_{2}-j_{2}\right] \bar{\phi}_{2}+\bar{\Theta}\right)\right\}
$$

We have thus achieved the main goal of this work, i.e., deriving the formal link between 
Møller operators and Miller's original SC-IVR expression of $S$ matrix elements in terms of shifted angles. In the next section, we derive an alternative and more natural semiclassical expression of $S$ matrix elements obtained by ignoring Møller operators. ${ }^{20}$ This expression, in natural angle representation, will be used to explain in relatively simple terms why and when Møller operators improve the efficiency of SC-IVR calculations. These developments will thus complement the analysis previously performed by Garashchuk and Light. We will also use the new representation to add a phase index to Eq. (72), crucial for an accurate description of interference effects in semiclassical methods based on the VVG propagator.

\section{$3 \quad$ SC-IVR in natural angles}

Rewriting Eq. (9) as

$$
S_{j_{2} j_{1}}=\int d \phi_{2} d \phi_{1}\left\langle j_{2} ; t \mid \phi_{2}\right\rangle\left\langle\phi_{2}\left|e^{-i H 2 t}\right| \phi_{1}\right\rangle\left\langle\phi_{1} \mid j_{1} ;-t\right\rangle
$$

and using Eqs. (6)-(8), (25) and (63) leads to

$$
\begin{aligned}
S_{j_{2} j_{1}}= & \left.\frac{1}{2 \pi(2 \pi i)^{1 / 2}} \int_{-\infty}^{+\infty} d \phi_{2} \int_{0}^{2 \pi} d \phi_{1} \sum_{\text {Paths }}\left|\frac{\partial \phi_{2}}{\partial J_{1}}\right|_{\phi_{1}}\right|^{-1 / 2} \\
& \quad \exp \left\{i\left(\left[j_{1}-J_{1}\right] \phi_{1}+\left[J_{2}-j_{2}\right] \phi_{2}+\Theta-\pi \eta / 2\right)\right\}
\end{aligned}
$$

with

$$
\Theta=\left(\frac{j_{2}^{2}+j_{1}^{2}}{2 I}\right) t-\int_{-t}^{t} d \tau(\phi \dot{J}+H)=\left(\frac{j_{2}^{2}+j_{1}^{2}}{2 I}\right) t-\int_{J_{1}}^{J_{2}} \phi d J-\int_{-t}^{t} d \tau H
$$

In Eq. (74), $\sum_{\text {Paths }}$ stands for $\sum_{k}$ in Eq. (25). Moreover, the indices $k$ in the phase and denominator of Eq. (25) are not explicitely written here. As previously, we integrate the RHS of this equation over $\phi_{1}$ within the SPA, i.e., by means of Eq. (A.11). Calling again $\varphi$ 
the phase of the integrand, we have

$$
\left.\frac{\partial \varphi}{\partial \phi_{1}}\right|_{\phi_{2}}=j_{1}-J_{1}-\left.\phi_{1} \frac{\partial J_{1}}{\partial \phi_{1}}\right|_{\phi_{2}}+\left.\phi_{2} \frac{\partial J_{2}}{\partial \phi_{1}}\right|_{\phi_{2}}+\left.\frac{\partial \Theta}{\partial \phi_{1}}\right|_{\phi_{2}}
$$

with from the RHS of Eq. (75),

$$
\left.\frac{\partial \Theta}{\partial \phi_{1}}\right|_{\phi_{2}}=-\left.\phi_{2} \frac{\partial J_{2}}{\partial \phi_{1}}\right|_{\phi_{2}}+\left.\phi_{1} \frac{\partial J_{1}}{\partial \phi_{1}}\right|_{\phi_{2}} .
$$

The two previous equations lead to

(a)

$$
\begin{aligned}
& \phi_{1}=\phi_{1}+\delta \phi_{1} \\
& J_{1}=J_{1}=0
\end{aligned}
$$

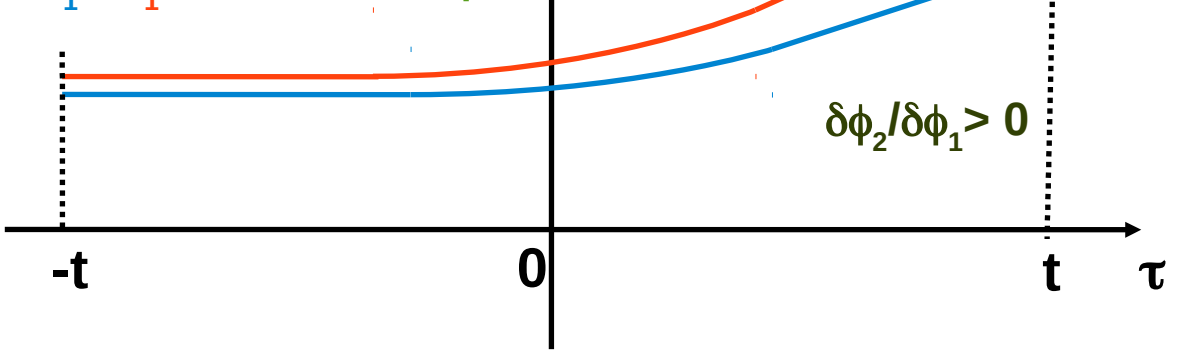

(b)

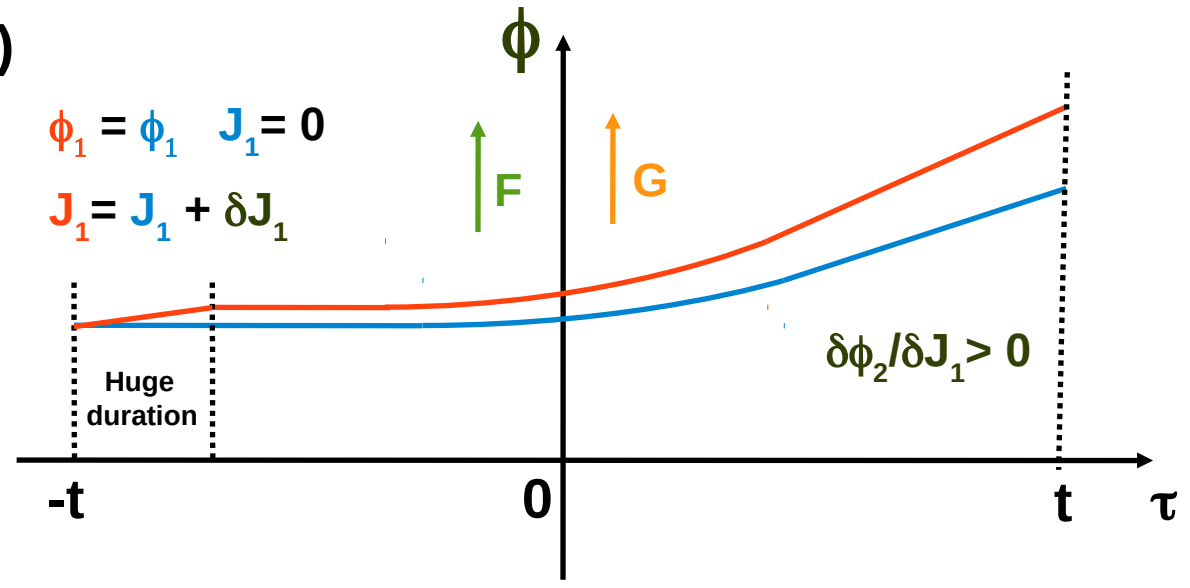

Figure 4: Examples of deflected paths starting with a very small difference $\delta \phi_{1}$ (a) or $\delta J_{1}$ (b). $\mathbf{F}$ is the force responsible for the deflection within the interaction region and $\mathbf{G}$ is its gradient. $\mathbf{F}$ and $\mathbf{G}$ are parallel (see text for more details). 
(a)

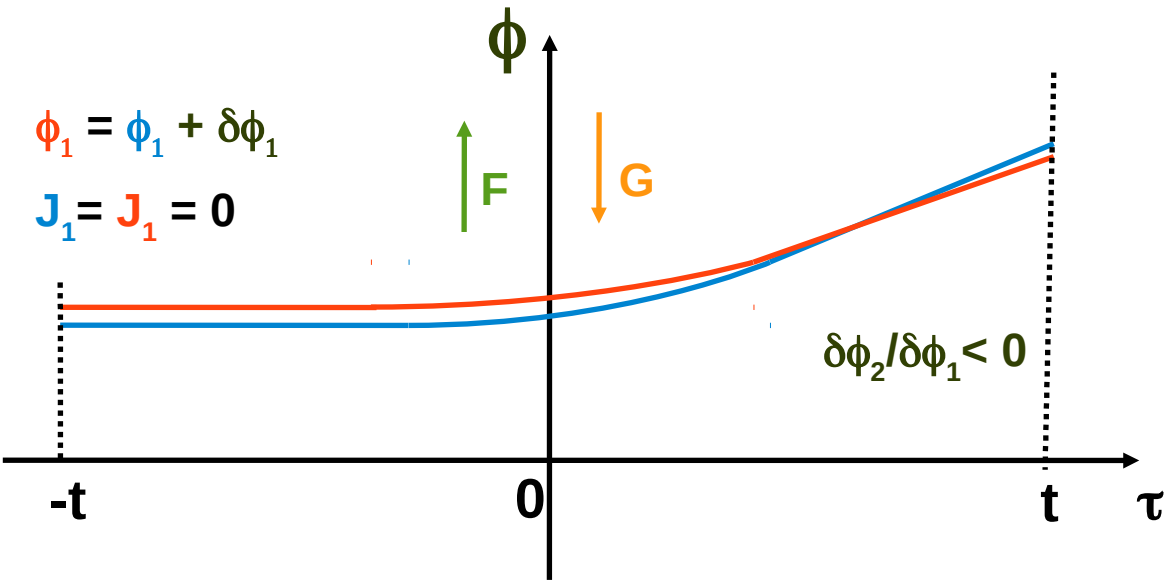

(b)

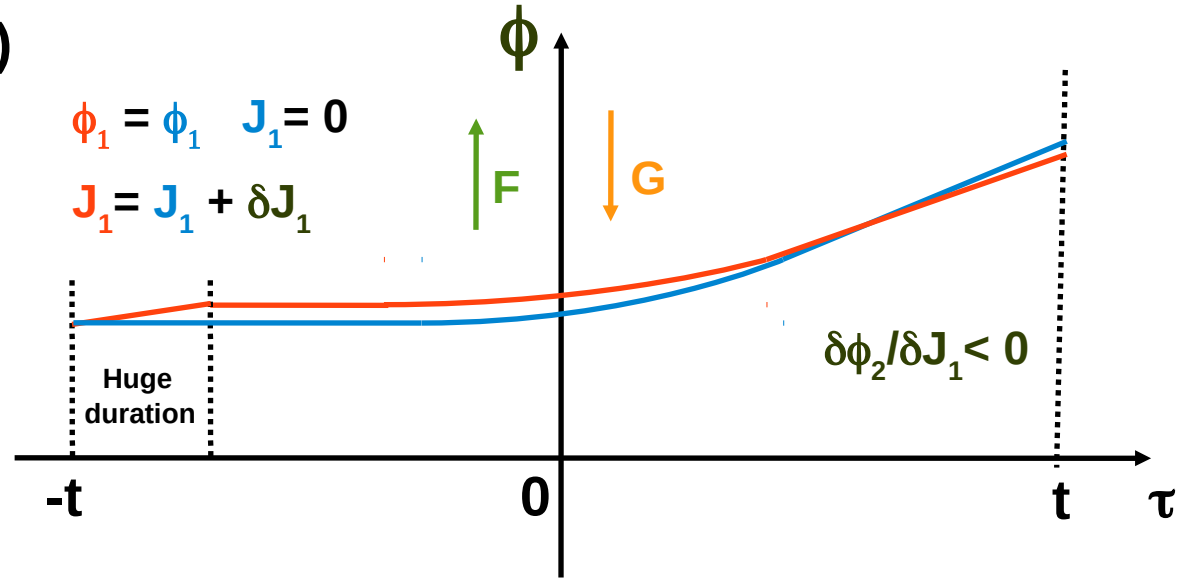

Figure 5: Examples of deflected paths starting with a very small difference $\delta \phi_{1}$ (a) or $\delta J_{1}$ (b). $\mathbf{F}$ is the force responsible for the deflection within the interaction region and $\mathbf{G}$ is its gradient. F and $\mathbf{G}$ are antiparallel (see text for more details).

$$
\left.\frac{\partial \varphi}{\partial \phi_{1}}\right|_{\phi_{2}}=j_{1}-J_{1}
$$

Thus, the values of $\phi_{1}$ making $\varphi$ stationary satisfy $J_{1}\left(\phi_{2}, \phi_{1}\right)=j_{1}$. Moreover,

$$
\frac{\partial^{2} \varphi}{\partial \phi_{1}^{2}}=-\left.\frac{\partial J_{1}}{\partial \phi_{1}}\right|_{\phi_{2}}=\left.\frac{\partial \phi_{2}}{\partial \phi_{1}}\right|_{J_{1}} /\left.\frac{\partial \phi_{2}}{\partial J_{1}}\right|_{\phi_{1}}
$$

The second equality is Eq. (42). Following Eq. (A.11), the phase index $\eta$ in Eq. (74) is increased by 0 (1) whenever $\frac{\partial^{2} \varphi}{\partial \phi_{1}^{2}}$ is positive (negative). Panel (a) in Fig. 4 shows a blue path starting from $\left(\phi_{1}, J_{1}=0\right)$ at $\tau=-t$. This path, assumed to make $\varphi$ stationary in the case 
where $j_{1}=0$, is then deflected up within the interaction region by the force $\mathbf{F}$ oriented along the $\phi$-axis. A second red path is run from $\left(\phi_{1}+\delta \phi_{1}, J_{1}=0\right)$ at $\tau=-t, \delta \phi_{1}$ being positive and infinitesimal. This path is thus slightly shifted up with respect to the blue path up to the entrance of the interaction region. Throughout the latter, the red path is more deflected than the blue path since the gradient $\mathbf{G}$ of $\mathbf{F}$ is supposed to be oriented parallel to $\mathbf{F}$. The red path, thus, deviates from the blue path and $\left.\frac{\partial \phi_{2}}{\partial \phi_{1}}\right|_{J_{1}}$ is positive at $\tau=t$. In panel (b), the blue path is the same as previously whereas the red path is run from $\left(\phi_{1}, J_{1}=\delta J_{1}\right)$ at $\tau=-t, \delta J_{1}$ being positive and infinitesimal. The period of time before entering the interaction region is supposed to be huge. However, $\delta J_{1}$ can be chosen as small as desired to make the blue and red paths parallel when they enter the interaction region, as in panel (a). The conclusion regarding the deviation of the red path from the blue one is thus the same as before and $\left.\frac{\partial \phi_{2}}{\partial J_{1}}\right|_{\phi_{1}}$ is positive at $\tau=t$. Since both $\left.\frac{\partial \phi_{2}}{\partial \phi_{1}}\right|_{J_{1}}$ and $\left.\frac{\partial \phi_{2}}{\partial J_{1}}\right|_{\phi_{1}}$ have the same sign, Eq. (79) shows that $\frac{\partial^{2} \varphi}{\partial \phi_{1}^{2}}$ is positive. The case where $\mathbf{G}$ and $\mathbf{F}$ are antiparallel is considered in Fig. 5, and the conclusion is the same as previously, i.e., both $\left.\frac{\partial \phi_{2}}{\partial \phi_{1}}\right|_{J_{1}}$ and $\left.\frac{\partial \phi_{2}}{\partial J_{1}}\right|_{\phi_{1}}$ are negative at $\tau=t$ (supposed here to be larger than the time at which the blue and red paths cross). Consequently, the SPA does not increase the phase index $\eta$. Using Eqs. (A.11), (79) and the fact that $j_{1}=J_{1}$, Eq. (74) becomes

$$
S_{j_{2} j_{1}}=\left.\frac{1}{2 \pi} \int_{-\infty}^{+\infty} d \phi_{2} \sum_{\text {Branches }}\left|\frac{\partial \phi_{2}}{\partial \phi_{1}}\right|_{J_{1}}\right|^{-1 / 2} \exp \left\{i\left(\left[J_{2}-j_{2}\right] \phi_{2}+\Theta-\pi \eta / 2\right)\right\}
$$

Proceeding as in Sec. 2.5 for the end of the derivation (see the developments following Eq. (71)), we finally obtain the SC-IVR expression in natural coordinates:

$$
S_{j_{2} j_{1}}=\left.\frac{1}{2 \pi} \int_{0}^{2 \pi} d \phi_{1}\left|\frac{\partial \phi_{2}}{\partial \phi_{1}}\right|_{J_{1}}\right|^{1 / 2} \exp \left\{i\left(\left[J_{2}-j_{2}\right] \phi_{2}+\Theta-\pi \eta / 2\right)\right\}
$$

The isomorphism between Eqs. (72) and (81) is stricking. So far, however, Eq. (72) involves no phase index. We thus add it in the next section. 


\section{Phase index for SC-IVR in shifted angles}

The complete SC-IVR form in shifted angles reads

$$
S_{j_{2} j_{1}}=\left.\frac{1}{2 \pi} \int_{0}^{2 \pi} d \bar{\phi}_{1}\left|\frac{\partial \bar{\phi}_{2}}{\partial \bar{\phi}_{1}}\right|_{J_{1}}\right|^{1 / 2} \exp \left\{i\left(\left[J_{2}-j_{2}\right] \bar{\phi}_{2}+\bar{\Theta}-\pi \bar{\nu} / 2\right)\right\}
$$

where $\bar{\Theta}$ is given by Eq. (65) with $J_{1}=j_{1}$, and $\bar{\nu}$ is the phase index to be determined. We still call $\varphi$ the phase of the integrand and integrate over $\bar{\phi}_{1}$ within the SPA, i.e., by means of Eq. (A.11). A few steps of algebra analogous to those following Eqs. (66) or (74) lead to

$$
\frac{\partial \varphi}{\partial \bar{\phi}_{1}}=\left.\left(J_{2}-j_{2}\right) \frac{\partial \bar{\phi}_{2}}{\partial \bar{\phi}_{1}}\right|_{J_{1}}
$$

and

$$
\frac{\partial^{2} \varphi}{\partial \bar{\phi}_{1}^{2}}=\left.\left.\frac{\partial J_{2}}{\partial \bar{\phi}_{1}}\right|_{J_{1}} \frac{\partial \bar{\phi}_{2}}{\partial \bar{\phi}_{1}}\right|_{J_{1}}+\left.\left(J_{2}-j_{2}\right) \frac{\partial^{2} \bar{\phi}_{2}}{\partial \bar{\phi}_{1}^{2}}\right|_{J_{1}}
$$

$\varphi$ is thus stationary when either $J_{2}=j_{2}$ or $\left.\frac{\partial \bar{\phi}_{2}}{\partial \bar{\phi}_{1}}\right|_{J_{1}}=0$ [we ignore the improbable case where both previous conditions are satisfied at the same time, or both $\left.\frac{\partial \bar{\phi}_{2}}{\partial \bar{\phi}_{1}}\right|_{J_{1}}$ and $\left.\frac{\partial^{2} \bar{\phi}_{2}}{\partial \bar{\phi}_{1}^{2}}\right|_{J_{1}}$ are zero at the same time, since this would make $\frac{\partial^{2} \varphi}{\partial \bar{\phi}_{1}^{2}}$ zero, and hence the SPA inapplicable (see Eq. (A.11))]. Now, trajectories for which $\left.\frac{\partial \bar{\phi}_{2}}{\partial \bar{\phi}_{1}}\right|_{J_{1}}$ is zero cannot contribute to $S_{j_{2} j_{1}}$, since the pre-exponential factor in Eq. (82) is $\left.\left|\frac{\partial \bar{\phi}_{2}}{\partial \bar{\phi}_{1}}\right|_{J_{1}}\right|^{1 / 2}$. Therefore, we are left with trajectories satisfying $J_{2}=j_{2}$. Then, Eqs. (82), (A.11) and (84) lead to the standard expression of classical $S$ matrix theory (CSMT) in shifted coordinates:

$$
S_{j_{2} j_{1}}=\sum_{k}\left[\frac{2 \pi}{i}\left|\frac{\partial J_{2}}{\partial \bar{\phi}_{1}}\right|_{J_{1}}^{k} \mid\right]^{-1 / 2} \exp \left\{i\left(\bar{\Theta}_{k}-\pi\left(\bar{\nu}_{k}+\bar{\chi}_{k}\right) / 2\right)\right\}
$$

where $\bar{\Theta}$ is now given by Eq. (65) with both $J_{1}=j_{1}$ and $J_{2}=j_{2}$ and the index $\bar{\chi}$ is equal to 0 (1) when $\left.\left.\frac{\partial J_{2}}{\partial \bar{\phi}_{1}}\right|_{J_{1}} \frac{\partial \bar{\phi}_{2}}{\partial \bar{\phi}_{1}}\right|_{J_{1}}$ is positive (negative). Similar developments allow to deduce from 
Eq. (81) the CSMT expression in natural coordinates:

$$
S_{j_{2} j_{1}}=\sum_{k}\left[\frac{2 \pi}{i}\left|\frac{\partial J_{2}}{\partial \phi_{1}}\right|_{J_{1}}^{k} \mid\right]^{-1 / 2} \exp \left\{i\left(\Theta_{k}-\pi \eta_{k} / 2\right)\right\}
$$

We recall that the Maslov index $\eta_{k}$ is equal to the number of times the blue path (the $k^{\text {th }}$ trajectory) and the red path in Figs. 4 and 5 cross each other between $-t$ and $t$, where $t$ is supposed to tend to $+\infty$ (see Eq. (9) and the line below it). Thus, $\eta_{k}$ is the number of times $\left.\frac{\partial \phi}{\partial J_{1}}\right|_{\phi_{1}}$, or $\left.\frac{\partial \phi}{\partial \phi_{1}}\right|_{J_{1}}$, takes the value 0 from infinite past to infinite future. We wish to emphasize that $\eta_{k}$ is generally defined as the number of times $\left.\frac{\partial \phi}{\partial J_{1}}\right|_{\phi_{1}}$ takes the value 0 , not $\left.\frac{\partial \phi}{\partial \phi_{1}}\right|_{J_{1}}$. But in the present case where $t$ tends to $+\infty$, one may indifferently use both derivatives.

In fact, Eq. (86) requires that $\left.\left.\frac{\partial J_{2}}{\partial \phi_{1}}\right|_{J_{1}} \frac{\partial \phi_{2}}{\partial \phi_{1}}\right|_{J_{1}}$ be always positive (for a reason similar to the one given at the end of the line following Eq. (85)). Otherwise, $\eta_{k}$ must be replaced by $\eta_{k}+1$ in Eq. (86). However, it is clear from the discussion of Figs. 4 and 5 that both $\left.\frac{\partial J_{2}}{\partial \phi_{1}}\right|_{J_{1}}$ and $\left.\frac{\partial \phi_{2}}{\partial \phi_{1}}\right|_{J_{1}}$ have the same sign at $\tau=t$, thus making $\left.\left.\frac{\partial J_{2}}{\partial \phi_{1}}\right|_{J_{1}} \frac{\partial \phi_{2}}{\partial \phi_{1}}\right|_{J_{1}}$ necessarily positive. One note that this is not the case in Fig. 5 between the entrance within the interaction region and the crossing point, for during this lapse of time, $\left.\frac{\partial J_{2}}{\partial \phi_{1}}\right|_{J_{1}}$ is negative (the two paths are approaching each other) while $\left.\frac{\partial \phi_{2}}{\partial \phi_{1}}\right|_{J_{1}}$ is positive. In other words, the correct value of the Maslov index is obtained on the right side of the crossing point of the blue and red paths. In practice, this may render the calculation of the index difficult when the crossing point occurs at an exceedingly large value of time.

In the classical limit (full integration within the SPA), all semiclassical formulations must lead to the same predictions. Eqs. (85) and (86) are thus necessarily equivalent. This is clear for the pre-exponential factors, as Eq. (36) implies $\partial /\left.\partial \bar{\phi}_{1}\right|_{J_{1}}=\partial /\left.\partial \phi_{1}\right|_{J_{1}}$. This is also clear for $\bar{\Theta}$ and $\Theta$ when comparing on one hand, Eq. (65) with both $J_{1}=j_{1}$ and $J_{2}=j_{2}$ and on the other hand, Eq. (75). Finally, this is also clear if the phase indices are identical, thus implying

$$
\bar{\nu}=\eta-\bar{\chi}
$$


This achieves the derivation of the complete SC-IVR form in shifted angles (Eqs. (82) and (87)). This derivation closely follows recent developments on the SC-IVR treatment of atomplanar rotor inelastic collisions in shifted coordinates. ${ }^{21,22}$

For practical purposes, Eq. (85) will be rewritten as

$$
S_{j_{2} j_{1}}=\int_{0}^{2 \pi} d \bar{\phi}_{1}\left[\frac{i}{2 \pi}\left|\frac{\partial J_{2}}{\partial \bar{\phi}_{1}}\right|_{J_{1}} \mid\right]^{1 / 2} \delta\left(J_{2}-j_{2}\right) \exp \{i(\bar{\Theta}-\pi \eta / 2)\}
$$

and the $\delta$ distribution will be replaced by the narrow Gaussian function

$$
G\left(J_{2}-j_{2}\right)=\exp \left[-\left(J_{2}-j_{2}\right)^{2} / \epsilon^{2}\right] /(\sqrt{\pi} \epsilon)
$$

with $\epsilon=0.01$, then making the Gaussian 0.017 wide. This is the Gaussian weighting, or binning implementation of CSMT. ${ }^{10,23}$ Eq. (86) could be rewritten in the same way.

\section{Effect of Møller operators on SC-IVR calculations: a case study}

In a first step, we apply Eqs. (81) and (82) to the system governed by the interaction potential

$$
V(\phi, \tau)=\alpha \cos ^{2} \phi \delta(\tau) .
$$

The delta function is used in place of the Gaussian pulse of Ref. ${ }^{16}$ so we will be able to analytically solve the dynamics both classical and quantum mechanically. The system is assumed to be in the initial state $j_{1}=0, \alpha$ is taken at 2.5 and $I$ at 1 (with this value of $I, \phi$ increases by 1 radian every unit time when $J=1$ ). We recall that $\phi_{1}$ and $J_{1}$ are the dynamical conditions at $-t$. Moreover, Eqs. (81) and (82) result from integrating Eqs. (66) and (74) within the SPA over $\phi_{1}$ and $\bar{\phi}_{1}$, respectively, which forces $J_{1}$ to be equal to $j_{1}=0$. 
Motion being free up to $\tau=0$, the value of $\phi$ at $\tau=0$ is also $\phi_{1}$. Since

$$
\dot{J}=-\frac{\partial V}{\partial \phi}=\alpha \sin (2 \phi) \delta(\tau)
$$

the final value of $J$ is

$$
J_{2}=\alpha \sin \left(2 \phi_{1}\right)
$$

Consequently,

$$
\phi_{2}=\phi_{1}+\frac{J_{2}}{I} t=\phi_{1}+\frac{\alpha t}{I} \sin \left(2 \phi_{1}\right)
$$

and

$$
\left.\frac{\partial \phi_{2}}{\partial \phi_{1}}\right|_{J_{1}}=1+\frac{2 \alpha t}{I} \cos \left(2 \phi_{1}\right)
$$

From Eqs. (75), (27) and (90), we obtain after some mathematical steps,

$$
\Theta=\left(\frac{j_{2}^{2}-\alpha^{2} \sin ^{2}\left(2 \phi_{1}\right)}{2 I}\right) t-\alpha \phi_{1} \sin \left(2 \phi_{1}\right)-\alpha \cos ^{2} \phi_{1}
$$

Using Eqs. (92)-(95), Eq. (81) finally reads

$$
\begin{gathered}
S_{j_{2} 0}=\frac{1}{2 \pi} \int_{0}^{2 \pi} d \phi_{1}\left|1+\frac{2 \alpha t}{I} \cos \left(2 \phi_{1}\right)\right|^{1 / 2} \\
\exp \left\{i\left(-j_{2} \phi_{1}-\alpha \cos ^{2} \phi_{1}+\frac{t}{2 I}\left[j_{2}-\alpha \sin \left(2 \phi_{1}\right)\right]^{2}-\pi \eta / 2\right)\right\} .
\end{gathered}
$$

The only difference between the paths represented in Figs. 4 and 5, and those involved in the present case, is that the smooth connection between initial and final straight lines in the former is made instantly at $\tau=0$ in the latter. It is thus clear from the upper panels of Figs. 4 and 5 that when $\left.\frac{\partial \phi_{2}}{\partial \phi_{1}}\right|_{J_{1}}$ is positive (negative), the blue and red trajectories dot not cross (do cross once) and $\eta=0$ (1). Besides, from Eq. (36) and the fact that $J_{1}=0$,

$$
\bar{\phi}_{1}=\phi_{1}
$$


Using this identity, Eq. (37), and the left equality of Eq. (93), we arrive at

$$
\bar{\phi}_{2}=\bar{\phi}_{1}
$$

Moreover, from Eq. (65), some developments involving Eqs. (27), (91) and (92) lead to

$$
\bar{\Theta}=-\alpha \bar{\phi}_{1} \sin \left(2 \bar{\phi}_{1}\right)-\alpha \cos ^{2} \bar{\phi}_{1}
$$

The phase index $\bar{\nu}$ in Eq. (82) is equal to $\bar{\chi}-\eta$ (see Eq. (87)). $\bar{\chi}$ is equal to 0 (1) when $\left.\left.\frac{\partial J_{2}}{\partial \bar{\phi}_{1}}\right|_{J_{1}} \frac{\partial \bar{\phi}_{2}}{\partial \bar{\phi}_{1}}\right|_{J_{1}}=\left.\frac{\partial J_{2}}{\partial \bar{\phi}_{1}}\right|_{J_{1}}$ is positive (negative) (Eq. (98) implies $\left.\frac{\partial \bar{\phi}_{2}}{\partial \bar{\phi}_{1}}\right|_{J_{1}}=1$ ). $\eta$ is the Maslov index from infinite past to infinite future (see a few lines below Eq. (86)). Hence, $\eta=0$ (1) when $\left.\frac{\partial \phi_{2}}{\partial \phi_{1}}\right|_{J_{1}}$ is positive (negative) at $t=+\infty$. Now, $\left.\frac{\partial J_{2}}{\partial \bar{\phi}_{1}}\right|_{J_{1}}=\left.\frac{\partial J_{2}}{\partial \phi_{1}}\right|_{J_{1}}$ (as implied by Eqs. (36) or (97)) and we have seen from Fig. 4 that $\left.\frac{\partial J_{2}}{\partial \phi_{1}}\right|_{J_{1}}$ and $\left.\frac{\partial \phi_{2}}{\partial \phi_{1}}\right|_{J_{1}}$ have the same sign at $t=+\infty$. Consequently, $\bar{\chi}$ and $\eta$ are equal and $\bar{\nu}$ is 0. Using this result, Eq. (92) and Eqs. (97)-(99), Eq. (82) finally reads

$$
S_{j_{2} 0}=\frac{1}{2 \pi} \int_{0}^{2 \pi} d \bar{\phi}_{1} \exp \left[-i\left(j_{2} \bar{\phi}_{1}+\alpha \cos ^{2} \bar{\phi}_{1}\right)\right]
$$

Lastly, the classical-limit quantum mechanical or CSMT expression of $S_{j_{2} 0}$ deduced from Eqs. (88), (89), (92) and (97) reads

$$
\begin{array}{r}
S_{j_{2} 0}=\int_{0}^{2 \pi} d \bar{\phi}_{1}\left[\alpha\left|\cos \left(2 \bar{\phi}_{1}\right)\right|\right]^{1 / 2} \exp \left[-\left(\alpha \sin \left(2 \bar{\phi}_{1}\right)-j_{2}\right)^{2} / \epsilon^{2}\right] /(\pi \epsilon) \\
\exp \{i(\bar{\Theta}-\pi \eta / 2+\pi / 4)\}
\end{array}
$$

with $\bar{\Theta}$ given by Eq. (99).

We now consider the quantum mechanical treatment. As shown in Appendix B, potential operators of the type $V_{0} \delta(\tau)$ lead to the evolution operator

$$
F=e^{-i H 2 t}=e^{-i H_{0} t} e^{-i V_{0}} e^{-i H_{0} t}
$$


known as Floquet operator. ${ }^{24}$ Here, the initial and final times are $-t$ and $t$, respectively, and $V_{0}=\alpha \cos ^{2} \phi$ (see Eq. (90)). Using Eqs. (6), (7), (9) and (102), one quickly arrives at the conclusion that the exact quantum mechanical expression of $S_{j_{2} 0}$ is also given by the RHS of Eq. (100). Remarkably, the system at hand belongs to a special class for which SC-IVR in shifted angles is thus exact. We note that Eq. (100) is independent on $t$, contrary to Eq. (96). Furthermore, one may easily show that the latter reduces to the former when $t$ tends to 0 provided that the Maslov index $\eta$ is calculated at $t=0$, not at $t=+\infty$. At time 0 , the blue and red paths are indeed infinitely short, so they cannot cross each other. Consequently, $\eta=0$. Now, Eq. (96) with both $t$ and $\eta$ taken at 0 is just Eq. (100). SC-IVR in shifted and natural angles will be called IVR-S and IVR-N, respectively. IVR-N calculations will be performed for a given value of $t$, and $\eta$ will be calculated for this value.

The IVR-S (or IVR-N at $t=0$ ) rotational state distribution $P_{j_{2}}=\left|S_{j_{2} 0}\right|^{2}$ is represented in Fig. 6 (magenta filled circles; lines are a guide for the eye). As in previous works, ${ }^{2,21}$ this distribution is converged with very few trajectories; in the present case, no more than 25 trajectories had to be run at equally spaced increments of $\bar{\phi}_{1}$. The CSMT, or classical-limit QM distribution, is represented by the light green filled circles. Here, this approach is of mild accuracy. It satisfyingly reproduces $P_{0}$ and the fact that odd transitions are prohibited, but not the populations $P_{ \pm 2}$ and $P_{ \pm 4}$ of the remaining available states. Quite interestingly, the IVR-N distribution is found to asymptotically converge towards the CSMT one as $t$ tends to infinity. Such a convergence, which does not seem to have been previously reported in the literature, strickingly appears in Fig. 7 where the values of $S_{00}, S_{ \pm 20}$ and $S_{ \pm 40}$ obtained from the previous semiclassical approaches are represented for different values of $t$. The lower right set of geometrical forms is associated with $S_{00}$, the left one with $S_{ \pm 20}$ and the upper right one with $S_{ \pm 40}$. The meaning of the different geometrical forms within these sets is as follows. Like in Fig. 6, magenta filled circles, or more precisely their centers, correspond to the IVR-S values of $S$-matrix elements (Eq. (100)), while light green filled circles correspond to their CSMT values (Eq. (101)). Since the CSMT value of $P_{ \pm 4}$ is 0 (see Fig. 6), the center 


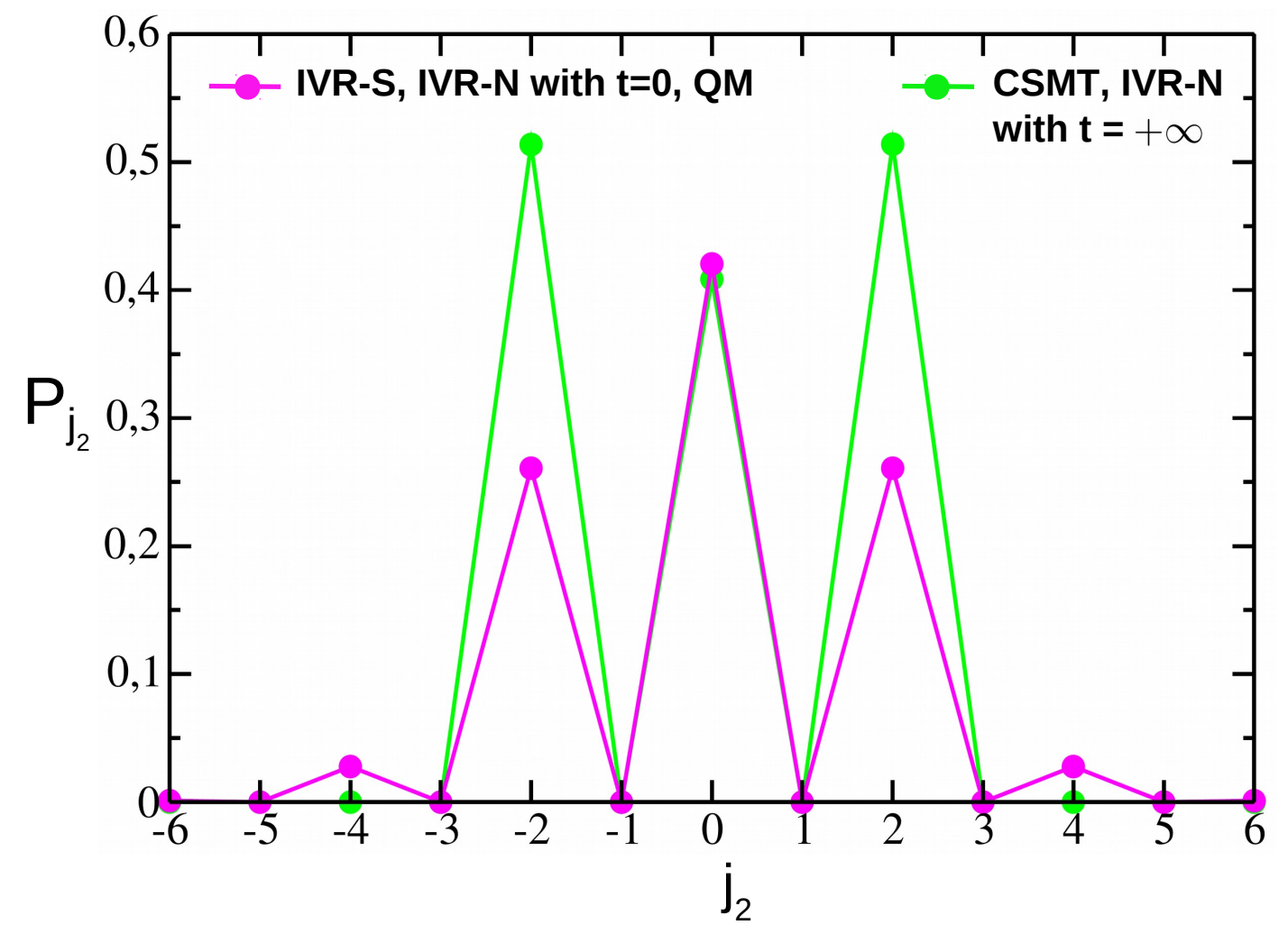

Figure 6: Rotational state distributions obtained from several approaches for the interaction potential $V(\phi, \tau)=\alpha \cos ^{2} \phi \delta(\tau)$. Magenta filled circles: IVR-S or IVR-N at $t=0$. This distribution is quantum mechanically exact (QM). Light green filled circles: CSMT or IVR-N at $t=+\infty$. The interaction potential being symmetric with respect to $\pi$, odd transitions are prohibited.

of the upper green circle in Fig. 7 lies at the origin of the complex plane. The white filled circles correspond to the IVR-N values (Eq. (96)) at $t=200$. If one had represented them at time 0 , these circles would have overlapped the magenta ones. But as time increases, white circles move anticlockwise away from magenta circles. For $j_{2}=0$ (lower right set), the path followed by the white circle between $t=0$ and 200 takes a spiral shape after a short transitory period (this curve is made of 20000 points spaced by 0.01 unit time). One clearly sees that (i) the center of this spiral is the green circle and (ii) the local radius of the spiral decreases as time goes on. Consequently, the white circle ovelaps the magenta 
circle at $t=+\infty$. For the two excited states, the scenario is similar, with the difference that the path followed by the white circle now results from a combination of trochoid and spiral shapes which gives it an air of nautilus shell cut in half. In summary, IVR-N is quantum mechanically exact at time 0 (for the system under scrutiny), and tends to classical-limit quantum scattering theory (CSMT) at an infinite time. ${ }^{25}$ The action of Møller operators has a very clear geometrical interpretation here: they make $S$-matrix elements run along the spirals (or mixed trochoid-spirals) from the white circles back to the magenta circles.

The convergence of IVR-N towards CSMT can also be easily proved mathematically in the case where $j_{2}=0$. As previously seen, the CSMT value of $S_{00}$ is due to trajectories starting at $-t$ with $J_{1}=0$ and arriving at $t$ with $J_{2}=0$. Eq. (92) shows that there are four trajectories defined by $\phi_{1}=0, \pi / 2, \pi$ and $3 \pi / 2$, satisfying these boundary conditions. Calling $\varphi$ the phase of Eq. (96) (forgetting the Maslov index), its second order development around $\phi_{1}=0$ and $\phi_{1}=\pi / 2$ leads to

$$
\varphi=\alpha\left(1+\frac{2 \alpha t}{I}\right) \phi_{1}^{2}-\alpha
$$

and

$$
\varphi=\alpha\left(\frac{2 \alpha t}{I}-1\right)\left(\phi_{1}-\frac{\pi}{2}\right)^{2}
$$

respectively. Analogous developments around $\phi_{1}=\pi$ and $\phi_{1}=3 \pi / 2$ lead to Eqs. (103) and (104) with $\phi_{1}-\pi$ and $\phi_{1}-3 \pi / 2$ instead of $\phi_{1}$ and $\phi_{1}-\pi / 2$. As expected, the four values of $\phi_{1}$ considered here make $\varphi$ stationary, in line with what has been seen in the previous section (see the passage from SC-IVR to CSMT). Eqs. (103) and (104) clearly show that the larger $t$, the larger the rate of oscillation of $\exp (i \varphi)$ when moving away from the stationary points, and the more valid the SPA. Therefore, as $t$ increases, IVR-N tends to CSMT. As far as the convergence of IVR-N results for a given value of $t$ is concerned, the minimum number of points necessary to accurately estimate them was found to be $\sim 25(1+2 t)$. Thus, this number linearly increases with $t$. 


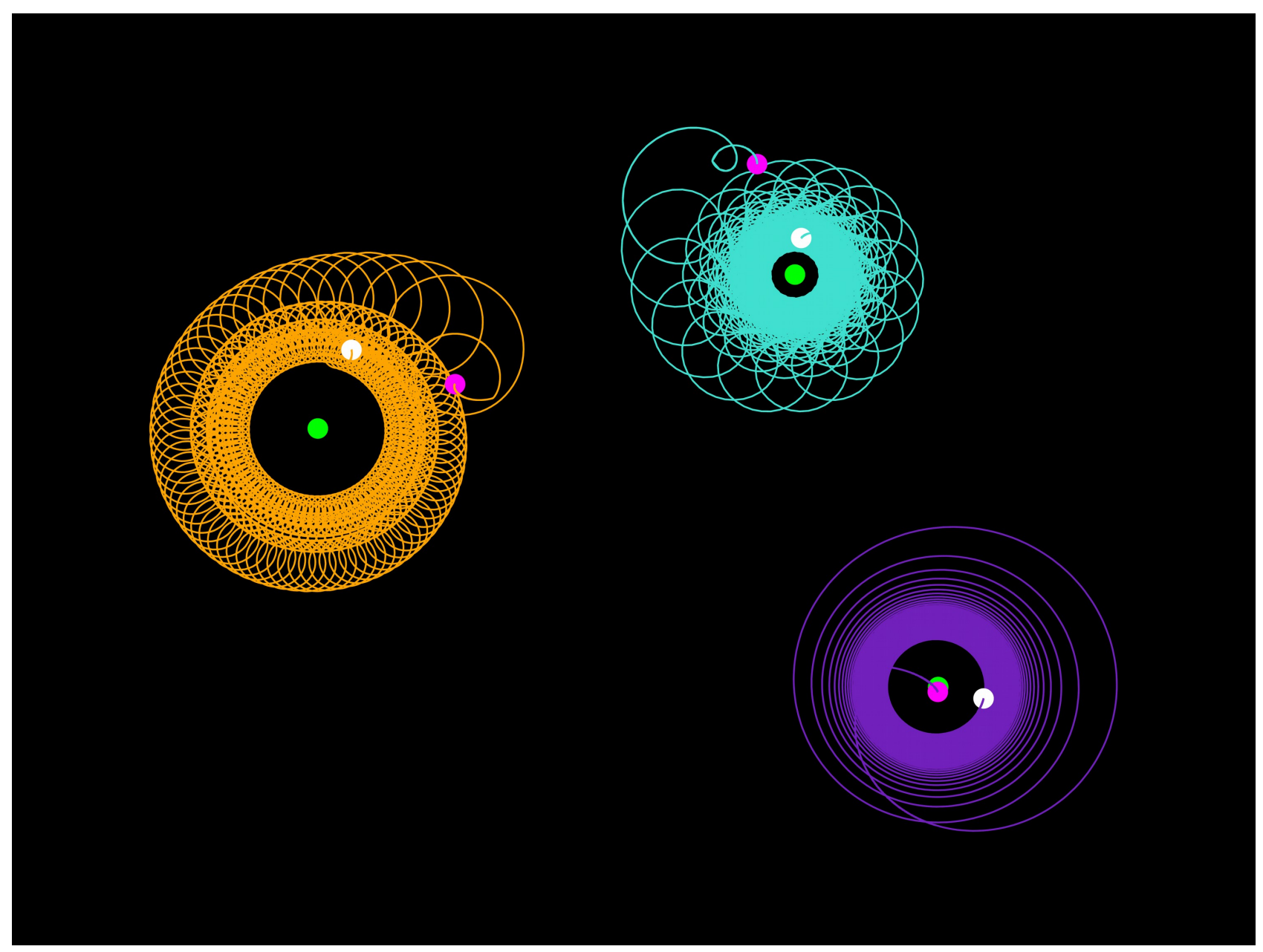

Figure 7: Representation of $S_{00}$ (lower right), $S_{ \pm 20}$ (left) and $S_{ \pm 40}$ (upper right) obtained from IVR-S or IVR-N at $t=0$ (centers of magenta filled circles), IVR-N at $t=200$ (centers of white filled circles), IVR-N from $t=0$ to 200 (lines going from the centers of magenta filled circles to the centers of white filled circles), and CSMT (centers of light green filled circles; the upper one lies at the origin of the complex plane). For $S_{00}$ (lower right), one clearly sees that as $t$ increases, the white circle runs along a spiral of which the center is the green circle and the radius decreases. Therefore, in the limit where $t$ tends to $+\infty$, the white circle overlaps the green one. In other words, IVR-N at $t=+\infty$ is equivalent to CSMT. For the remaining elements, white circles run along more complex paths of mixed trochoid-spiral shapes but the final conclusion is the same. The action of Møller operators amounts to run the white circles along the previous paths back to the magenta circles. For the present system, magenta circles give the exact quantum mechanical values of $S$-matrix elements (see text for more details).

To sum up, over the range of small values of $t$ making IVR-N calculations non timeconsuming, IVR-N results strongly vary around IVR-S ones in terms of $t$. Consequently, if one randomly chose a given value of $t$ within the previous range, as one would do in 
actual calculations dealing with a realistic non instantaneous interaction $(t$ would then be the duration between the exit of the interaction region and the stop of the trajectories), one is very likely to get quite inaccurate IVR-N predictions. Moreover, IVR-N converges towards CSMT in the limit where $t$ tends to $+\infty$, which has two major flaws: calculations are indeed all the more time-consuming as $t$ grows, and they are potentially inaccurate (like $P_{ \pm 2}$ ). In contrast, IVR-S does not suffer from the same drawbacks since the action of Møller operators kills extra oscillations due to the dynamics outside the interaction region, making thereby the predictions not only independent on $t$, but also much more accurate ${ }^{21}$ (exact for the system at hand). Last but not least, one might think that the full-dimensional expression of $S_{j_{2} j_{1}}$ in natural coordinates, given by Eqs. (74) and (75), would better behave as $t$ increases, but we have numerically checked that this not the case. Whenever Møller operators are not used, this expression also leads to predictions converging towards CSMT ones as the period of time spent by the system outside the interaction region increases (the numerical estimation of Eq. (74) involves the substitution of $d \phi_{2}$ by $\left|\frac{\partial \phi_{2}}{\partial J_{1}}\right|_{\phi_{1}} \mid d J_{1}$ and the integration over $\phi_{1}$ and $\left.J_{1}\right)$.

We have also considered the case of a non instantaneous interaction by replacing $\delta(\tau)$ in Eq. (90) by the Gaussian function

$$
G(\tau)=\exp \left(-\tau^{2} / \epsilon^{2}\right) /(\sqrt{\pi} \epsilon)
$$

with $\epsilon=0.3$. For this value, one may consider that the system crosses the exit of the interaction region at $t=0.9$. This value of $t$ is thus the minimum time at which one can estimate the final outcomes (it is the analog of 0 in the previous case). The rotational state distributions obtained by means of IVR-S and IVR-N at $t=0.9$ are compared with the exact QM distribution in Fig. 8. The QM calculations are detailed in Appendix C. The IVR-S distribution appears to be in much better agreement with the QM distribution than the IVR-N one. The norm $\sum_{j_{2}} P_{j_{2}}$ of the former is 0.9828 , very close to the exact 


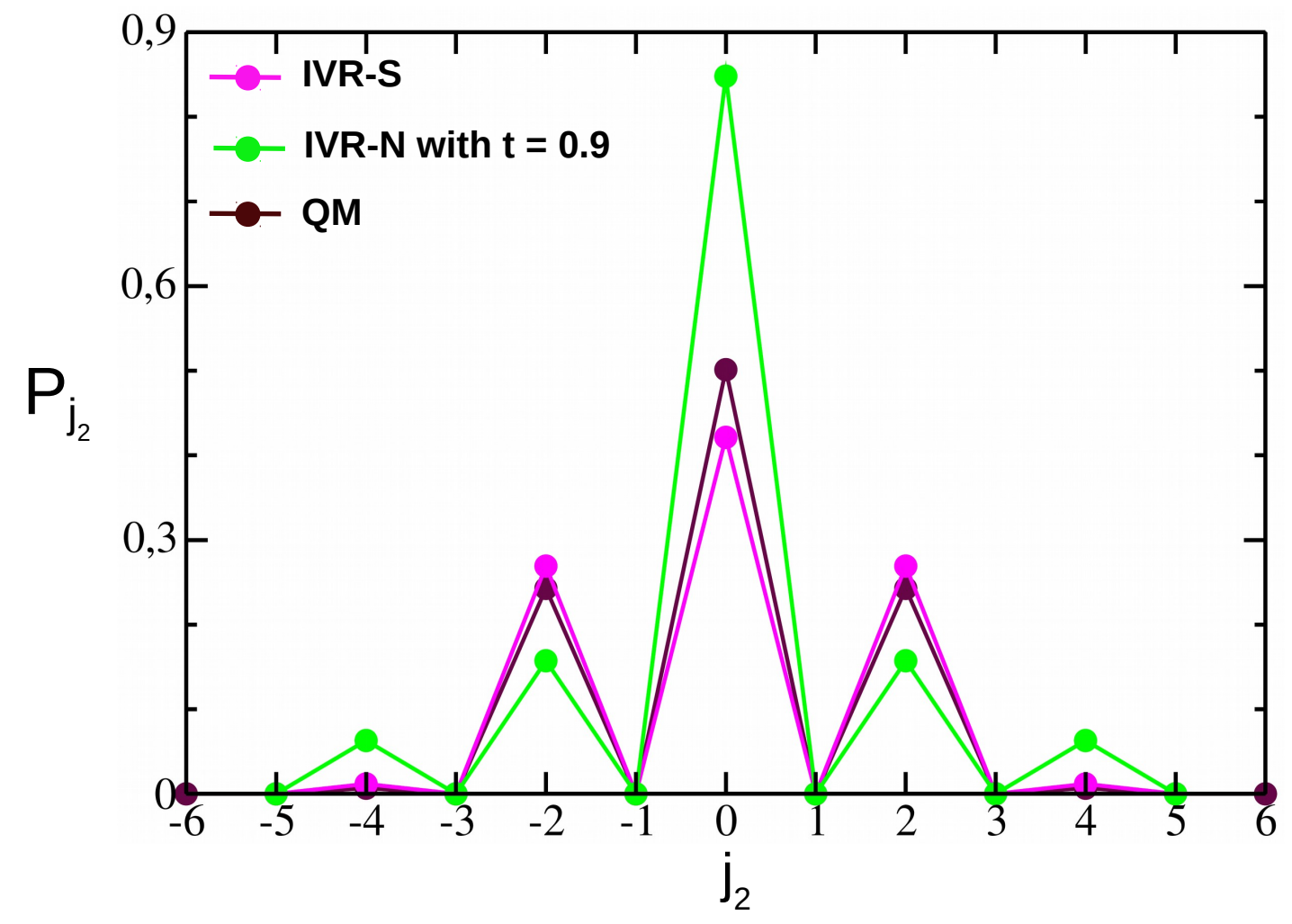

Figure 8: Rotational state distributions obtained from several approaches for the interaction potential $V(\phi, \tau)=\alpha \cos ^{2} \phi \exp \left(-\tau^{2} / \epsilon^{2}\right) /(\sqrt{\pi} \epsilon)$ with $\epsilon=0.3$. Magenta filled circles: IVR-S. Black filled circles: QM. Light green filled circles: IVR-N at $t=0.9$, corresponding to the exit of the interaction region.

unit norm, against 1.2885 for the latter. Moreover, the IVR-S distribution requires only 25 trajectories to be converged, against $\sim 250$ for the IVR-N distribution. Lastly, we have numerically checked that the IVR-N distribution converges towards the CSMT one as $t$ tends to infinity. Therefore, if the beginning of the scenario is not the same as before (equivalence of IVR-S and IVR-N at $t=0$ ), it ends in the same way. We note that in contrast with the previous case, the best IVR-N results, at $t=0.9$, are only of mild accuracy as compared with the IVR-S ones. Moreover, they require ten times more trajectories to be converged, in line with Garashchuk and Light's results on the collinear $\mathrm{H}+\mathrm{H}_{2}$ reaction. ${ }^{17}$ It 
is worth pointing out that if Møller operators strongly decrease the amount of trajectories required to converge vibrational state populations in the previous process, they leave these populations unchanged. This is in contrast with the present results which show that Møller operators not only reduce the required amount of trajectories but also increase the accuracy of rotational state populations. The reasons behind such differences are still unknown and need further studies to be found. We also note that the quality of the IVR-S predictions has deteriorated slightly as compared to the previous case, and we have checked that the larger $\epsilon$, i.e. the duration of the interaction, the worst the agreement between IVR-S and QM distributions (not to mention IVR-N ones). This disagreement might be reduced by using more sophisticated semiclassical propagators, like the Herman-Kluck one, ${ }^{5,6,9}$ but as is well established, non linearities in the interaction potential make any semiclassical propagator inaccurate in the long-time limit.

\section{Conclusion}

The semiclassical initial value representation (SC-IVR) was originally introduced by Miller ${ }^{14}$ in the context of molecular scattering ${ }^{5,13}$ to go round the root search issue of classical $S$ matrix theory (CSMT). ${ }^{1}$ The central result of his derivation, formula (29) in Ref. ${ }^{14}$, improved not only the feasibility of semiclassical scattering calculations, but also their accuracy. ${ }^{13}$

This formula involves classical trajectory runs which are the classical analogs of Møller operators, known to play a fundamental role in quantum scattering theory. ${ }^{5,13}$ Nevertheless, these operators do not appear in Miller's developments. In fact, three decades passed before Møller operators were explicitely introduced by Garashchuk and Light ${ }^{17}$ in SC-IVR calculations performed in Cartesian coordinates within the Tannor and Weeks formulation of quantum scattering theory. ${ }^{18}$

In the present paper, we have revisited Miller's formulation within a simple model of light-induced rotational transitions so as to make Møller operators appear at the begining of 
its derivation. We have then recovered formula (29) in Ref. ${ }^{14}$ by using the stationary phase approximation $^{4}$ and juggling with partial derivatives in order to express them in terms of Miller's angular coordinates, ${ }^{14}$ which have the property to be shifted with respect to those commonly used in Hamilton equations. Thereby, we have clearly demonstrated that Møller operators were already underlying Miller's pioneering derivation.

A first example of instantaneous interaction was considered, for which Miller-type predictions are quantum mechanically exact. SC-IVR predictions ignoring Møller operators are also found to be exact provided that trajectories spend an infinitely short period of time outside the (infinitely narrow) interaction region. However, contrary to Miller-type predictions, they degrade as the period of time spent by trajectories outside the interaction region increases, with the additional disadvantage that they require more and more trajectories to be converged. Last but not least, Møller-free SC-IVR tends to classical-limit quantum scattering theory (CSMT) when the previous period of time tends to infinity.

A second example of non instantaneous interaction was studied, for which Miller-type results are in much better agreement with quantum results than Møller-free SC-IVR results obtained at the exit of the interaction region. Moreover, the former results require ten times less trajectories than the latter, thereby corroborating Garashchuk and Light's results on the $\mathrm{H}+\mathrm{H}_{2}$ reaction. ${ }^{17}$ Just as in the previous case, Møller-free SC-IVR tends to CSMT as the period of time spent by the trajectories outside the interaction region increases, and more and more trajectories are necessary.

For all such reasons, it is highly recommended to follow Miller, ${ }^{14}$ Garashchuk and Light ${ }^{17}$ and use Møller operators in future SC-IVR scattering calculations. This should be of particular importance for accurate semiclassical treatments of rotational transitions which might have to be derived in non action-angle coordinates for improving their realism. 


\section{Acknowledgements}

I am grateful to the two reviewers of this paper, one for allowing me to better understand how IVR-N converges towards CSMT as $t$ tends to infinity (which led to Fig. 7), and the other for pointing out the exactness of Eq. (100). 


\section{Appendix A: Semiclassical space-time propagation of the free rotor}

For the free rotor considered in this work,

$$
\phi_{b}=\phi_{a}+\frac{J_{a}}{I} t
$$

where $J_{a}$ is the value at $t_{a}$ of the rotational angular momentum conjugate to $\phi$. Consequently,

$$
\left.\frac{\partial \phi_{b}}{\partial J_{a}}\right|_{\phi_{a}}=\frac{t}{I}
$$

Moreover, the Lagrangien reduces to the rotational energy which, here, is a constant of motion. Therefore, the action integral (see Eq. (19)) is given by

$$
R\left(\phi_{b}, \phi_{a} ; t\right)=\int_{t_{a}}^{t_{b}=t_{a}+t} d \tau \frac{J^{2}}{2 I}=\frac{J_{a}^{2}}{2 I} t .
$$

Using Eqs. (A.1) and (A.3) allows to express the action in terms of the angles and time:

$$
R\left(\phi_{b}, \phi_{a} ; t\right)=\frac{I\left(\phi_{b}-\phi_{a}\right)^{2}}{2 t}
$$

Using Eq. (18) and noting from Eq. (A.2) that the Maslov index is necessarily $0\left(\left.\frac{\partial \phi}{\partial J_{a}}\right|_{\phi_{a}}=\frac{\tau-t_{a}}{I}\right.$ never takes the value 0 beyond $t_{a}$ ), we arrive at

$$
\left\langle\phi_{b}\left|e^{-i H_{0} t}\right| \phi_{a}\right\rangle=\left(\frac{I}{2 \pi i t}\right)^{1 / 2} e^{i\left(\frac{I\left(\phi_{b}-\phi_{a}\right)^{2}}{2 t}\right)} .
$$

For a free particle of coordinate $x$ and mass $m$, the right-hand-side (RHS) of this equation with $\left(x_{b}, x_{a}, m\right)$ substituted to $\left(\phi_{b}, \phi_{a}, I\right)$ is quantum mechanically exact. But for our free rotor, the problem is more subtle. We will indeed deduce from the next developments that 
the time-evolved rotational state

$$
\left\langle\phi_{b} \mid j ; t\right\rangle=\int_{0}^{2 \pi} d \phi_{a}\left\langle\phi_{b}\left|e^{-i H_{0} t}\right| \phi_{a}\right\rangle\left\langle\phi_{a} \mid j ; 0\right\rangle
$$

obtained using Eq. (A.5) is incorrect.

The discussion preceding Eq. (20) emphasizes the necessity to take into account in the semiclassical propagation all the classical paths going from $\left(\phi_{a}, t_{a}\right)$ to $\left(\phi_{b}+2 k \pi, t_{b}\right)$, whatever the value of $k$, as they all reach the same physical configuration of the system. This can be done by replacing Eq. (A.6) by

$$
\left\langle\phi_{b} \mid j ; t\right\rangle=\sum_{k=-\infty}^{+\infty} \int_{0}^{2 \pi} d \phi_{a}\left\langle\phi_{b}+2 \pi k\left|e^{-i H_{0} t}\right| \phi_{a}\right\rangle\left\langle\phi_{a} \mid j ; 0\right\rangle
$$

We now check that Eqs. (A.5) and (A.7) correctly propagate $\left\langle\phi_{a} \mid j ; 0\right\rangle$, i.e., are consistent with Eq. (7). Using Eqs. (A.5), (A.7) and (6), we have

$$
\left\langle\phi_{b} \mid j ; t\right\rangle=\frac{1}{(2 \pi)^{1 / 2}}\left(\frac{I}{2 \pi i t}\right)^{1 / 2} \sum_{k=-\infty}^{+\infty} \int_{0}^{2 \pi} d \phi_{a} e^{i\left[\frac{I\left(\phi_{b}+2 \pi k-\phi_{a}\right)^{2}}{2 t}+j \phi_{a}\right]} .
$$

Making the change of variable $\phi=\phi_{a}-2 \pi k$ leads to

$$
\left\langle\phi_{b} \mid j ; t\right\rangle=\frac{1}{(2 \pi)^{1 / 2}}\left(\frac{I}{2 \pi i t}\right)^{1 / 2} \sum_{k=-\infty}^{+\infty} \int_{-2 \pi k}^{2 \pi(1-k)} d \phi e^{i\left[\frac{I\left(\phi_{b}-\phi\right)^{2}}{2 t}+j \phi+2 \pi j k\right]}
$$

or equivalently,

$$
\left\langle\phi_{b} \mid j ; t\right\rangle=\frac{1}{(2 \pi)^{1 / 2}}\left(\frac{I}{2 \pi i t}\right)^{1 / 2} \int_{-\infty}^{+\infty} d \phi e^{i\left[\frac{I\left(\phi_{b}-\phi\right)^{2}}{2 t}+j \phi\right]}
$$

Integration over $\phi$ can be exactly performed by using the central result of the SPA:

$$
\int_{-\infty}^{+\infty} d \phi g(\phi) e^{i f(\phi)}=\sum_{k} g\left(\phi_{k}\right)\left[\frac{2 \pi i}{\left|f^{\prime \prime}\left(\phi_{k}\right)\right|}\right]^{1 / 2} e^{i\left[f\left(\phi_{k}\right)-\pi \nu_{k} / 2\right]}
$$


$\phi_{k}$ is the $k^{t h}$ stationary point of $f$ and $\nu_{k}$ is equal to $0(1)$ if $f^{\prime \prime}\left(\phi_{k}\right)$ is positive (negative). Here,

$$
g(\phi)=1
$$

and

$$
f(\phi)=\frac{I\left(\phi_{b}-\phi\right)^{2}}{2 t}+j \phi
$$

so $f^{\prime}(\phi)=0$ implies the single stationary point

$$
\phi=\phi_{b}-\frac{j}{I} t
$$

Moreover,

$$
f^{\prime \prime}(\phi)=\frac{I}{t}
$$

So

$$
\nu=0
$$

Inserting Eq. (A.14) in Eq. (A.13) leads to

$$
f(\phi)=-\frac{j^{2}}{2 I} t+j \phi_{b}=-E_{j} t+j \phi_{b}
$$

(see Eq. (8)). The integral in Eq. (A.10) is thus given by the RHS of Eq. (A.11) together with Eqs. (A.12) and (A.15)-(A.17). Substituting the resulting expression to the integral in Eq. (A.10) finally leads to the expected result

$$
\left\langle\phi_{b} \mid j ; t\right\rangle=e^{-i E_{j} t}\left\langle\phi_{b} \mid j ; 0\right\rangle
$$

(see Eq. (7)). The previous integration is exact since $g(\phi)$ is constant and $f(\phi)$ is quadratic (see Eqs. (A.12) and (A.13)). 
Note that instead of using Eqs. (A.5) and (A.7), we might have used Eq. (A.6) and

$$
\left\langle\phi_{b}\left|e^{-i H_{0} t}\right| \phi_{a}\right\rangle=\sum_{k=-\infty}^{+\infty}\left(\frac{I}{2 \pi i t}\right)^{1 / 2} e^{i\left(\frac{I\left(\phi_{b}+2 \pi k-\phi_{a}\right)^{2}}{2 t}\right)} .
$$

However, we chose the previous expressions in order to keep in line with Eqs. (20) and (23)-(25)

\section{Appendix B: Floquet operator}

The developments of this appendix follow the presentation of Floquet operators by Stockmann. ${ }^{24}$ We consider the evolution operator $e^{-i H 2 t}$ applied at time $\tau=-t$ to a given quantum state, $H$ being given by Eq. (4). Without loss of generality, $e^{-i H 2 t}$ can be rewritten as

$$
F=e^{-i H(t-\Delta / 2)} e^{-i H \Delta} e^{-i H(t-\Delta / 2)}
$$

Now, we assume that the interaction occurs instantaneously at time $\tau=0$. Thus, the potential energy operator reads

$$
V=V_{0} \delta(\tau)
$$

Making $\Delta$ tend to $0^{+}$allows to rewrite $V$ as

$$
V=V_{0} / \Delta
$$

for $\tau$ within the range $]-\Delta / 2, \Delta / 2[$, and 0 outside this range. Hence, Eqs. (4) and (B.1) give

$$
F=e^{-i H_{0}(t-\Delta / 2)} e^{-i\left(H_{0} \Delta+V_{0}\right)} e^{-i H_{0}(t-\Delta / 2)} .
$$

Replacing $\Delta$ by 0 in this expression leads to Eq. (102). 


\section{Appendix C: Quantum calculations}

We consider the following wave-packet at a negative value of $\tau$ around $-t$ :

$$
\Psi(\phi, \tau)=\sum_{k} a_{k} \frac{e^{i\left(k \phi-E_{k} \tau\right)}}{(2 \pi)^{1 / 2}}
$$

with

$$
a_{k}=\delta_{k 0}
$$

At $-t$, this wave-packet represents the initial state $\langle\phi \mid 0 ;-t\rangle$ (see Eqs. (6) and (7)) of Eq. (9). Substituting the RHS of Eq. (C.1) to $\Psi$ in the Schrödinger equation

$$
i \frac{d \Psi}{d \tau}=-\frac{1}{2 I} \frac{\partial^{2} \Psi}{\partial \phi^{2}}+V \Psi
$$

with

$$
V=\alpha \cos ^{2} \phi G(\tau)
$$

$(G(\tau)$ is given by Eq. (105)) and projecting $\langle l ;-\tau \mid \phi\rangle$ on the left side of the resulting equation, we obtain

$$
\frac{d a_{l}^{r e}}{d \tau}=\sum_{k}\left(V_{l k}^{r e} a_{k}^{i m}+V_{l k}^{i m} a_{k}^{r e}\right)
$$

and

$$
\frac{d a_{l}^{i m}}{d \tau}=\sum_{k}\left(V_{l k}^{i m} a_{k}^{i m}-V_{l k}^{r e} a_{k}^{r e}\right) .
$$

The upperscripts $r e$ and $i m$ denote the real and imaginary parts of $a_{l}$ and $V_{l k}$. The latter is given by

$$
V_{l k}=\langle l ; \tau|V| k ; \tau\rangle=\frac{\alpha G(\tau)}{2}\left[\delta_{l k}+\frac{1}{2}\left(\delta_{(l-2) k}+\delta_{(l+2) k}\right)\right] e^{i\left(E_{l}-E_{k}\right) t}
$$

Use of the identities $\cos ^{2} x=[1+\cos (2 x)] / 2$ and $\cos x=\left(e^{i x}+e^{-i x}\right) / 2$ was made to derive this expression. 21 states ranging from $l=-10$ to 10 were considered. The fourth-order Runge-Kutta integrator ${ }^{26}$ was used to solve Eqs. (C.5) and (C.6) with a time step of 3/10 ${ }^{4}$ 


\section{Data Availability Statements}

The data that support the findings of this study are available from the corresponding author upon reasonable request.

\section{References}

(1) Miller, W. H. Classical-limit quantum mechanics and the theory of molecular collisions. Adv. Chem. Phys. 1974, 25, 69.

(2) McCurdy, C. W.; Miller, W. H. Interference effects in rotational state distributions: Propensity and inverse propensity. J. Chem. Phys. 1977, 67, 463.

(3) Kreek, H.; Marcus, R. A. Semiclassical collision theory: Multidimensional integral method. J. Chem. Phys. 1974, 61, 3308.

(4) Gutzwiller, M. C. Chaos in Classical and Quantum Mechanics; Springer-Verlag, New York, 1990.

(5) Tannor, D. J. Introduction to quantum mechanics. A time-dependent perspective; University Science Books, 2007.

(6) Heller, E. J. The Semiclassical Way to Dynamics and Spectroscopy; Princeton University Press, 2018.

(7) Liberto, G. D.; Ceotto, M. The importance of the pre-exponential factor in semiclassical molecular dynamics. J. Chem. Phys. 2016, 145, 144107.

(8) Miller, W. H. The semiclassical initial value representation: A potentially practical way for adding quantum effects to classical molecular dynamics simulations. J. Phys. Chem. A 2001, 105, 2942.

(9) Kay, K. Semiclassical initial value treatments of atoms and molecules. Annu. Rev. Phys. Chem. 2005, 56, 255. 
(10) Bonnet, L. Classical dynamics of chemical reactions in a quantum spirit. Int. Rev. Phys. Chem. 2013, 32, 171.

(11) Bonnet, L.; Espinosa-Garcia, J. Simulation of the experimental imaging results for the $\mathrm{OH}+\mathrm{CHD}_{3}$ reaction with a simple and accurate theoretical approach. Phys. Chem. Chem. Phys. 2017, 19, 20267.

(12) Bouakline, F.; Althorpe, S. C.; Larregaray, P.; Bonnet, L. Strong geometric-phase effects in the hydrogen-exchange reaction at high collision energies: II. Quasiclassical trajectory analysis. Mol. Phys. 2010, 108, 969-980.

(13) Zhang, J. Z. H. Theory and application of quantum molecular dynamics; World Scientific Publishing Company, 1999.

(14) Miller, W. H. Classical S Matrix: Numerical Application to Inelastic Collisions. J. Chem. Phys. 1970, 53, 3578.

(15) Skodje, R. T. On the use of the interaction picture in classical mechanics. Chem. Phys. Lett. 1984, 109, 221.

(16) Arango, C. A.; Kennerly, W. W.; Ezra, G. S. Semiclassical IVR approach to rotational excitation of non-polar diatomic molecules by non-resonant laser pulses. Chem. Phys. Lett. 2006, 420, 296.

(17) Garashchuk, S.; Light, J. C. Semiclassical application of the Møller operators in reactive scattering. J. Chem. Phys. 2001, 114, 1060.

(18) Tannor, D. J.; Weeks, D. E. Wave packet correlation function formulation of scattering theory: The quantum analog of classical Smatrix theory. J. Chem. Phys. 1993, 98, 3884 .

(19) Tabor, M. Chaos and Integrability in Non linear Dynamics; John Wiley and Sons, New York, 1989. 
(20) Bonnet, L.; Crespos, C. Phase-index problem in the semiclassical description of molecular collisions. Phys. Rev. A 2008, 78, 062713.

(21) Bonnet, L. Semiclassical initial value theory of rotationally inelastic scattering: Some remarks on the phase index in the interaction picture. J. Chem. Phys. 2018, 148, 194104.

(22) Miller's SC-IVR in shifted angles was called SC-IVR in the interaction picture ${ }^{13}$ (IP) in Ref. ${ }^{21}$ owing to the proximity between Møller operators and the IP.

(23) Sierra, J. D.; Bonnet, L.; Gonzalez, M. Quasi-Classical Trajectory-Gaussian Binning Study of the $\mathrm{OH}+\mathrm{D}_{2} \longrightarrow \operatorname{HOD}\left(\mathrm{v}_{1}{ }^{\prime}, \mathrm{v}_{2}{ }^{\prime}, \mathrm{v}_{3}{ }^{\prime}\right)+\mathrm{D}$ Angle-Velocity and Vibrational Distributions at a Collision Energy of $0.28 \mathrm{eV}$. J. Phys. Chem. A 2011, 115, 7413.

(24) Stockmann, H.-J. Quantum Chaos. An Introduction; Cambridge University Press, New York, 1999.

(25) Analogously, we observed a few years ago that for an inelastic collision between an atom and a planar rotator, IVR-N predictions tends towards CSMT ones whenever the initial and final radial distances are taken at infinity (unpublished work).

(26) William, H.; Teukolsky, S. A.; Vetterling, W. T.; Flannery, B. P. Numerical Recipes: The Art of Scientific Computing, 3rd ed.; Cambridge University Press, New York, 2007. 